University of South Florida

DIGITAL COMMONS

Digital Commons @ University of

@ UNIVERSITY OF SOUTH FLORIDA

South Florida

USF Tampa Graduate Theses and Dissertations

USF Graduate Theses and Dissertations

10-26-2016

\title{
Methodologies to Exploit ATPG Tools for De-camouflaging
}

Deepak Reddy Vontela

University of South Florida, deepakreddy@mail.usf.edu

Follow this and additional works at: https://digitalcommons.usf.edu/etd

Part of the Computer Engineering Commons

\section{Scholar Commons Citation}

Vontela, Deepak Reddy, "Methodologies to Exploit ATPG Tools for De-camouflaging" (2016). USF Tampa Graduate Theses and Dissertations.

https://digitalcommons.usf.edu/etd/6597

This Thesis is brought to you for free and open access by the USF Graduate Theses and Dissertations at Digital Commons @ University of South Florida. It has been accepted for inclusion in USF Tampa Graduate Theses and Dissertations by an authorized administrator of Digital Commons @ University of South Florida. For more information, please contact digitalcommons@usf.edu. 
Methodologies to Exploit ATPG Tools for De-camouflaging

by

Deepak Reddy Vontela

A thesis submitted in partial fulfillment

of the requirements for the degree of

Master of Science in Computer Engineering

Department of Computer Science and Engineering

College of Engineering

University of South Florida

Co-Major Professor: Swaroop Ghosh, Ph.D.

Co-Major Professor: Srinivas Katkoori, Ph.D.

Sriram Chellappan, Ph.D.

Date of Approval:

July 27, 2016

Keywords: Tetramax, Reverse engineering (RE),Controllability, Observability

Copyright ( 2016, Deepak Reddy Vontela 


\section{DEDICATION}

I would like to dedicate this thesis to my family and my friends who stood by my side and encouraging me throughout my master's. 


\section{ACKNOWLEDGMENTS}

I would like to give thanks to my co-major professors, Dr. Swaroop Ghosh, Dr. Srinivas Katkoori. I would be eternally thankful to Dr. Swaroop Ghosh, for providing opportunity to be part of his research group. His continuous guidance and dedication has helped me excel in my field of study for the past 2 years. I would like to acknowledge Dr. Sriram Challeppan, for being part of my thesis committee. I would like to thank my colleagues in the LOGICS Research laboratory, Anirudh Iyengar, Hamid Motaman, Jae-Won Jang and Rekha Govindraj for providing a great experience in the lab. I would like to give a special thanks to, Anirudh Iyengar, for providing an amazing foundation of work in camouflaged gate design. I would also like to express my gratitude to Hamid Motaman, Jae-Won Jang for guiding me in designing layout. 


\section{TABLE OF CONTENTS}

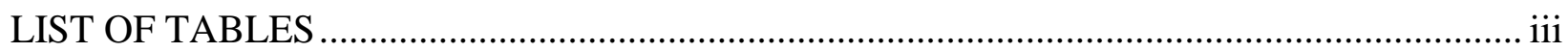

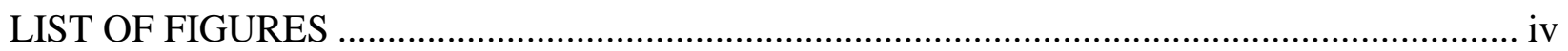

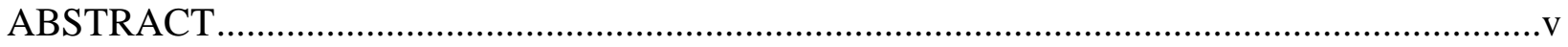

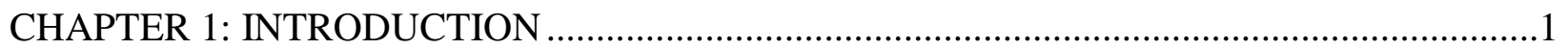

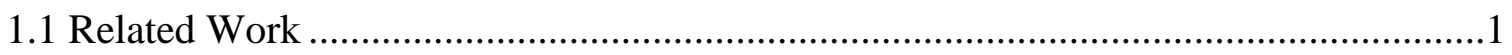

CHAPTER 2: AUTOMATIC TEST PATTERN GENERATION TOOL …………....................5

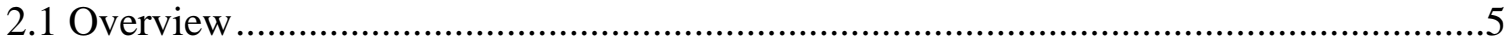

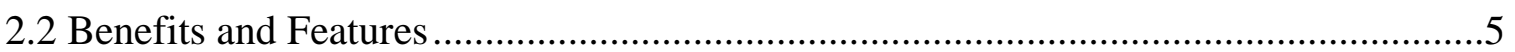

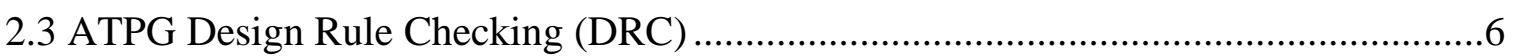

2.4 Formats Supported by Tetramax............................................................................

2.5 Challenges Faced Using Tetramax for RE..............................................................

CHAPTER 3: REVERSE ENGINEERING METHODLOGY USING ATPG TOOLS..................9

3.1 Gate Level Input Pattern Identification.....................................................................

3.1.1 Minimizing the Number of Gate Level Input Patterns ..................................

3.1.2 Methodologies to Exploit Stuck-at Fault Model............................................11

3.2 Netlist Level Test Pattern Generation Using ATPG............................................... 11

3.2.1 Dummy Gate Insertion.............................................................................11

3.2.2 Fault Sensitization and Propagation...............................................................12

3.2.3 Split and Stitch Methodology ……........................................................13

3.3 Reverse Engineering Effort....................................................................................14

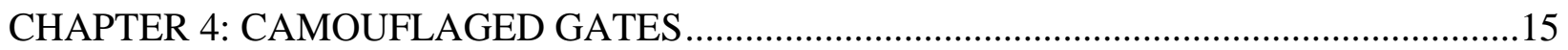

4.1 Threshold Voltage Defined Switch Design ............................................................15

4.2 Proposed Camouflage Gate....................................................................................16

4.3 Threshold Voltage Defined Multiplexer .................................................................18

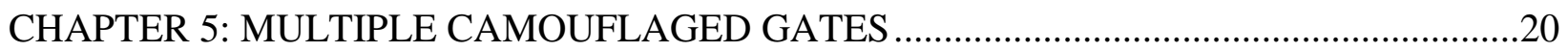

$5.1 \mathrm{RE}$ of Two Isolated Camouflaged Gates ………………………………………...20

5.2 RE of Two Series Connected Camouflaged Gates …………………………………..21

5.3 RE of Two Camouflaged Gates with a Known Gate in Between................................22

5.4 Three Series Connected Camouflaged Gates...............................................................23

5.5 Camouflaging Using Threshold Defined Multiplexer ………………........................23

5.5.1 Obfuscation Methodology …………………........................................24

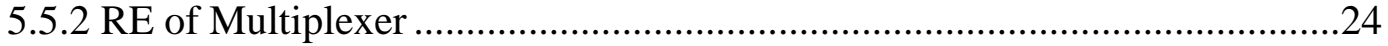




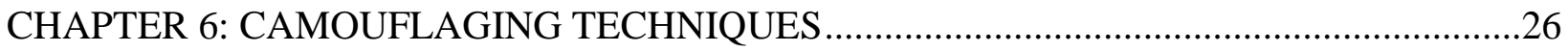

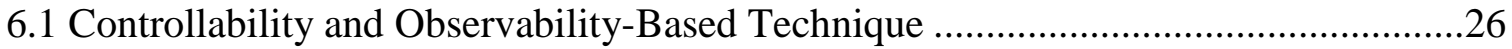

6.2 Hamming Distance Sensitivity-Based Technique

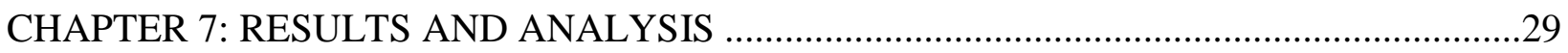

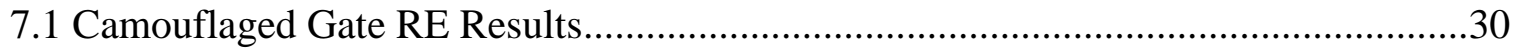

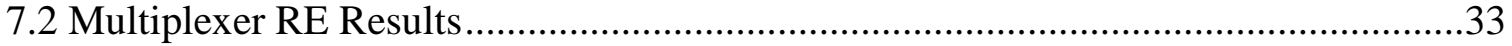

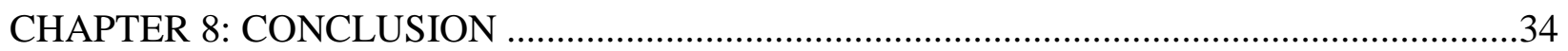

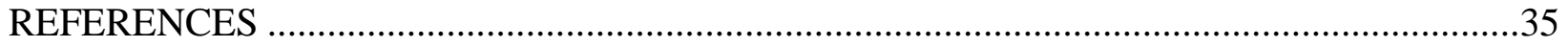

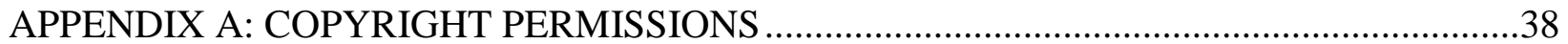




\section{LIST OF TABLES}

Table 3.1 Test Patterns for Different Logic Gates ..............................................................10

Table 3.2 Patterns Required to RE each Logic Function of Camouflaged Gate .........................10

Table 3.3 Gate Level Patterns and Corresponding Dummy Gate Types ..................................12

Table 4.1 Different Logic Implementation of Camouflage Gate ...........................................18

Table 6.1 Controllability and Observability Calculations for Two Input Gates........................26 


\section{LIST OF FIGURES}

Figure 2.1 Integrated Test Flow using Tetramax ATPG ..............................................................6

Figure 2.2 Tetramax Tool Showing C17 Benchmark Circuit ........................................................

Figure 3.1 De-camouflaging a Gate in C17 using Split and Stitch Method .................................13

Figure 4.1 PMOS/NMOS Switch ON/OFF Demonstration with Gate Voltage Vg .......................16

Figure 4.2 Camouflaged Gate with VT Defined Switches ………………...............................17

Figure 4.3 Schematic and Layout of Threshold Defined 2-1 Multiplexer......................................19

Figure 5.1 C17 Benchmark with Two Independent Camouflaged Gates ....................................20

Figure 5.2 C17 Benchmark with Series Connected Camouflaged Gates ....................................21

Figure 5.3 A Known Gate in Between Two Camouflaged Gates................................................22

Figure 5.4 Three Series Connected Camouflaged Gates ............................................................23

Figure 5.5 Number of Input Patterns vs Number of Series Connected Camouflaged Gates.........24

Figure 5.6 C17 Benchmark after Replacing the Selected Nets by Multiplexers ..........................25

Figure 6.1 Controllability and Observability Formulae Implementation ......................................27

Figure 7.1 Breakdown of RE Effort for HTRE with 5\% Camouflaging .....................................29

Figure 7.2 RE Effort for Different Benchmarks using HTCO...................................................30

Figure 7.3 RE Effort for HDS with Independent and 2-Series Dependent Gates..........................31

Figure 7.4 RE Effort for HTRE with Pure Gate Selection and 2-Series Dependent Gates ............32

Figure 7.5 Comparison of RE Effort with Various Flavors of HTCO, HDS and HTRE ...............32

Figure 7.6 RE Effort of Different Benchmarks Replaced with 2-1 Multiplexer ...........................33

Figure 7.7 RE Effort for Different Types Multiplexers for 5\% Camouflaging ...............................33 


\begin{abstract}
Semiconductor supply chain is increasingly getting exposed to Reverse Engineering (RE) of Intellectual Property (IP). Camouflaging of gates in integrated circuits are typically employed to hide the gate functionality to prevent reverse engineering. The functionalities of these gates cannot be found by De-layering as they don’t leave any layout clues. Adversaries perform reverse engineering by replacing the camouflaged gate with the known gate and by developing custom software to determine test patterns. These test patterns are used to analyze the outputs and to conclude the functionality of the camouflaged gate.

In this thesis, we show that reverse engineering of camouflaged design can be performed by exploiting the test features of commercial/publicly available Automatic Test Pattern Generation (ATPG) tools. We also propose controllability/observability and Hamming Distance sensitivity based metric to select target gates for camouflaging. Simulations on ISCAS85 benchmarks shows that the proposed techniques can increase the reverse engineering effort significantly by camouflaging small fraction of gates.
\end{abstract}




\section{CHAPTER 1}

\section{INTRODUCTION $^{1}$}

Reverse engineering of an IC is a process of identifying its structure, design and functionality [1]. Reverse engineering of IC's is performed to collect competitive intelligence and device technology used in the IC. This process is also used to check for any commercial piracy and patent infringements. There are many techniques and tools to enable reverse engineering of an IC. On the other side, reverse engineering has several benefits for adversary but these are threats to semiconductor industry such as he can use this to steal the IP and functionality of design. Once the functionality is known, similar devices can be fabricated. Adversary can use the components that are extracted from original products. Because of the above reasons reverse engineering(RE) is a serious threat to semiconductor industry [8].

\subsection{Related Work}

In the existing reverse engineering method, the adversary delayers the IC, determines the gate functionalities and their connectivity information, and, reconstructs the netlist. Split manufacturing [12] has been proposed to hide the interconnection between gates to discourage meaningful Trojan insertion. Camouflaging of gates is proposed to hide the logic functionality and make the reverse engineering impossible or extremely hard or economically unviable. It has been shown that careful camouflaging with $\sim 10-40 \%$ overhead can increase the reverse engineering effort significantly [19]. A camouflaged gate that can exhibit multiple functionalities will increase the reverse engineering effort. A camouflaged gate using dummy contacts [1] can realize 3

\footnotetext{
${ }^{1}$ This chapter was previously published in [1]. Permissions are included in Appendix A.
} 
functions at the cost of $\sim 5 \mathrm{X}$ area and power overhead. Programmable standard cells using control signals have also been proposed [14]. However, it requires signal routing for each camouflaged gate. Techniques to deceive the attacker using filler cells [15] and dummy transistors [16] are also proposed. The reverse engineering effort can also be increased by selecting appropriate gates in the netlist for camouflaging. It has been demonstrated that hard-to-control and hard-to-observe gates are typically good choices for camouflaging. Another metric is Hamming Distance (HD) sensitivity of the gate which determines the number of primary outputs (POs) that it can corrupt. More HD sensitivity infers higher reverse engineering effort since it increases the number of POs the adversary has to validate after each test-pattern application. Although these basic concepts have been presented in [1,19] an aggregate metric to select gates for camouflaging and its impact on reverse engineering effort has not been described. The conventional reverse engineering methodologies [20] rely on developing tools to read the partial netlist, generate/apply carefully derived test patterns (for a target camouflaged gate) on a fresh chip, observe the outputs and match with netlist's response to de-camouflage the target gate. This methodology is expensive and could be used only by adversaries with expertise in test pattern generation algorithms and programming. We show that a less-expensive reverse engineering methodology can be used by non-experts who have access to commercial or publicly available Automatic Test Pattern Generation (ATPG) tools to reverse engineering the camouflaged gates. However, exploiting ATPG tools and fault models to generate desired test patterns targeted to reverse engineering a gate is nontrivial process. This is true because the functionality of camouflaged gate is unknown which makes reading of the netlist by the tool impossible. Furthermore, the netlist-level pattern that is generated to apply a gate-level pattern to a target gate may not propagate the gate output to the PO. To the best of our knowledge this is the first effort on exploiting ATPG tools (Tetramax [21]) for reverse engineering. 
In this thesis we propose metrics to select gates for camouflaging and compare their impact on reverse engineering effort. We also propose an ATPG-aided comprehensive methodology to reverse engineering a netlist. For camouflaging we consider the gate proposed in [22] that can exhibit 6 functionalities. However, the proposed metrics and reverse engineering effort analysis is generic and can be extended to other flavors of camouflaged gates as well. There are two main aspects in the proposed reverse engineering process: (a) gate-level test pattern identification; and, (b) netlist-level test pattern generation. In the first part we find the minimum number of gate-level patterns from which the functionality of the camouflaged gate can be identified. This step is largely independent of the netlist and depends on the camouflaged gate or camouflaged function. In the netlist level test generation, we use Tetramax to generate patterns to ensure that the desired pattern is applied on the camouflaged gate and the effect could be observed at the PO. Test pattern generation is done by exploiting stuck-at fault models at the target camouflaged gate. The camouflaged gate is replaced with a pre-determined known gate to enable compilation of netlist by ATPG tool. The proposed methodology is applicable to single as well as multiple camouflaged gates.

This thesis provides an in-depth analysis of reverse engineering using ATPG tools and also designed of a multiplexer which is used to camouflage the interconnects. In summary, we make following contributions in this thesis,

- We propose a novel RE technique using stuck-at fault model of Tetramax commercial ATPG tool.

- We determine the RE effort of the above technique through detailed analysis.

- We provide treatment of both isolated as well as dependent set of camouflaged gates in the netlist. 
- We propose controllability, observability and HD sensitivity based metrics of the gates to select the target gates for camouflaging.

- We evaluate the strength of proposed camouflaging metrics using RE effort of the proposed RE methodology.

- We propose a multiplexer which is used to camouflage the interconnects.

- We designed a circuit of ring oscillators with the proposed camouflaged gate [22] for test chip simulations.

The rest of thesis is organized as follows. In Chapter II we discuss about ATPG tool its features and challenges we faced using the tool for RE. We provide a detailed analysis of the proposed RE methodology for single gate in Chapter III. Consideration for multiple camouflaged gates is presented in Chapter IV. The metrics for camouflaging is described in Chapter V. Chapter VI provides simulation results and conclusions are drawn in Chapter VII. 


\section{CHAPTER 2}

\section{AUTOMATIC TEST PATTERN GENERATION TOOL}

In this chapter we first introduce the ATPG tool its benefits and features and how we use this tool in RE and also the challenges we faced.

\subsection{Overview}

ATPG (Automatic Test Pattern Generation) is an electronic design automation technology used to find the input sequence [10]. This sequence when applied to a digital circuit enables test equipment to differentiate between the correct and faulty circuit behavior caused by the defects. The patterns generated are used to test semiconductor devices after manufacture to check the functionality and analyze the cause of failure if any. The effectiveness of ATPG is measured by the amount of detected faults and number of patterns generated. These metrics generally indicate test quality and test application time. ATPG efficiency is another important consideration, which is influenced by the type of circuit under test, level of abstraction and the required test quality.

\subsection{Benefits and Features}

Tetramax is the only ATPG tool with is integrated with DFTMAX and DFTMAXultrs [11]. It is the solution for wide range of test methodologies. Some of the key benefits and features:

- It can generate test patterns for most complex designs.

- It is faster because it isolates the defect locations.

- High performance and capacity.

- It supports multicore and run time.

- It has scan and design rule check(DRC). 


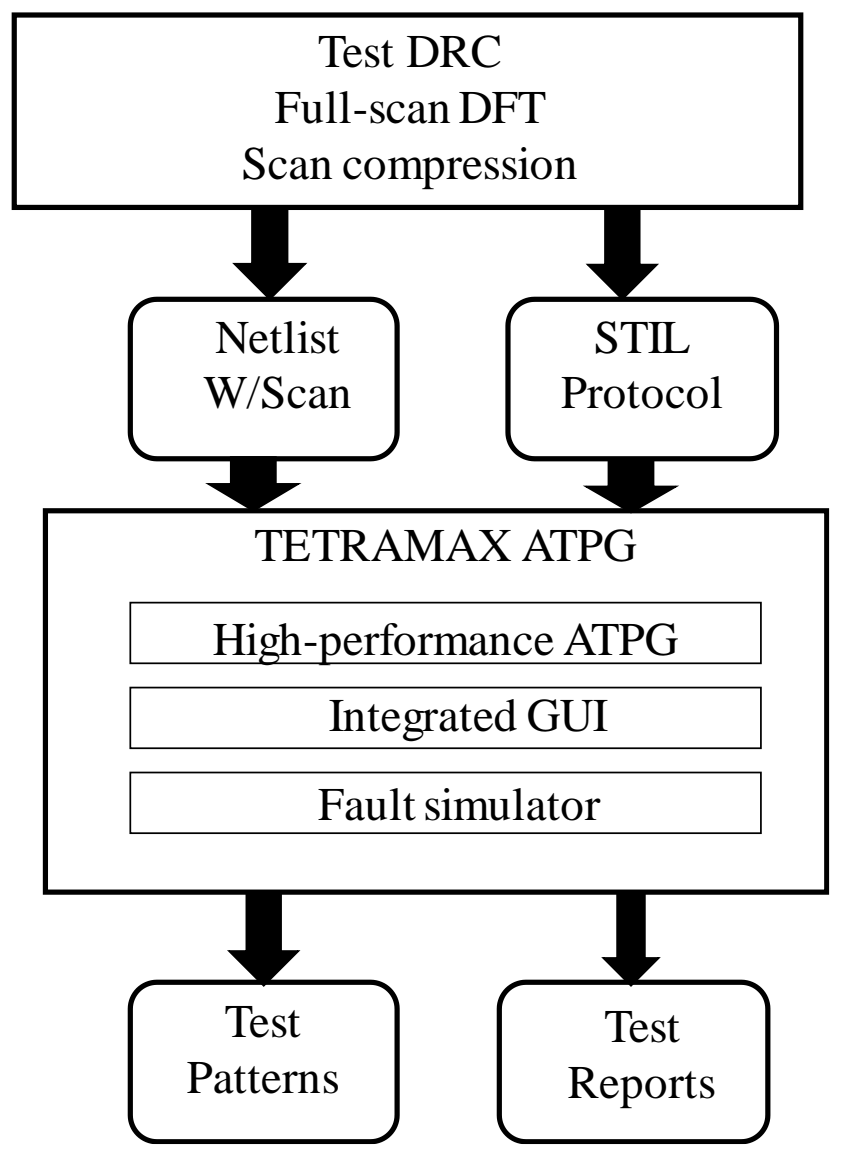

Figure 2.1 Integrated Test Flow using Tetramax ATPG

\subsection{ATPG Design Rule Checking (DRC)}

To run any kind of functionality in ATPG tool the basic step that the design should pass is the Design Rule Checking [12]. By running DRC we can identify the chip level test issues. Errors can be viewed directly on the circuit using graphical schematic viewer as shown in Fig 2.2. 


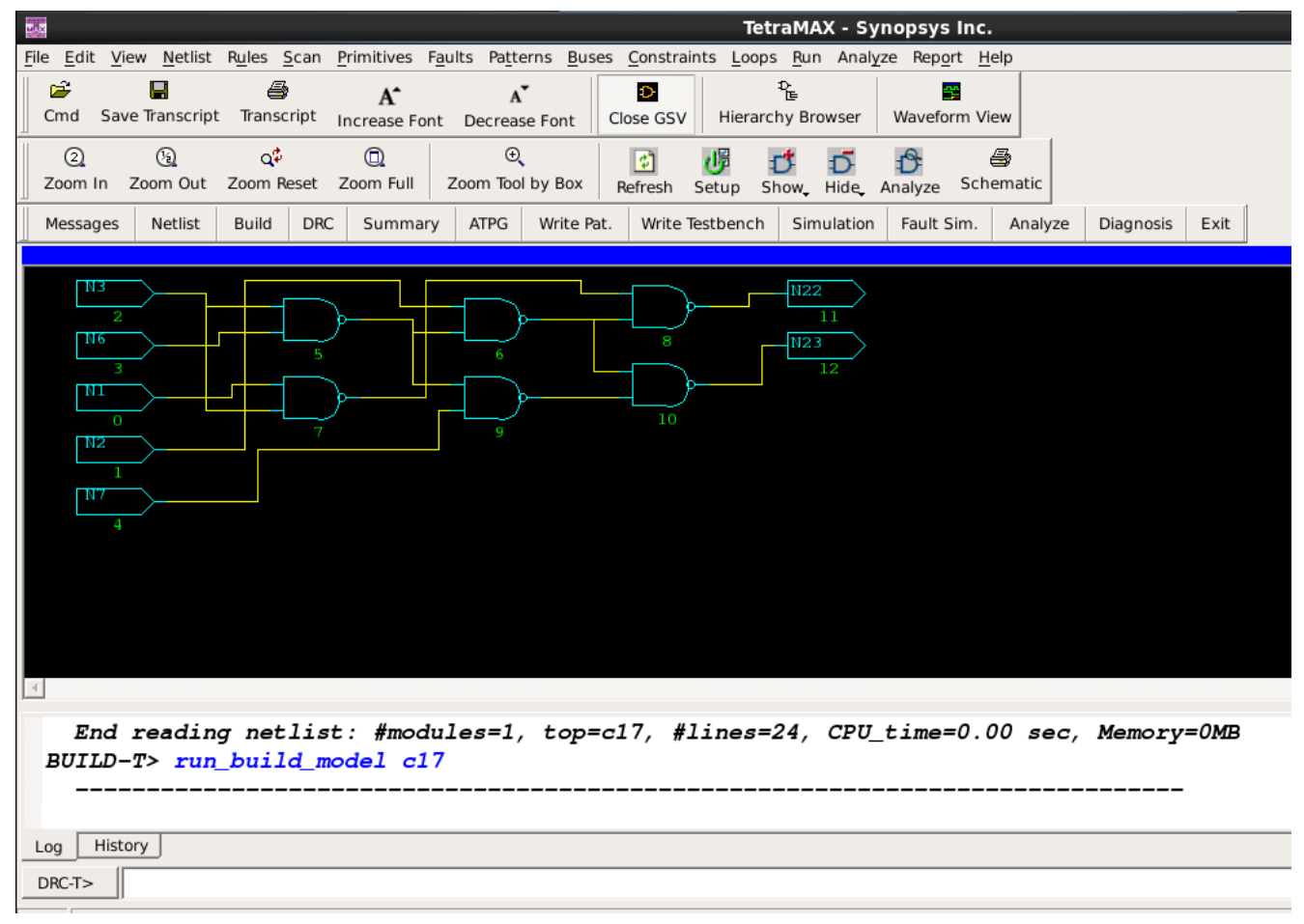

Figure 2.2 Tetramax Tool Showing C17 Benchmark Circuit.

\subsection{Formats Supported by Tetramax}

Tetramax supports many industrial formats namely circuit netlists like Verilog and VHDL,

SDC in timing, in layout it supports LEF/DEF and test patterns like STIL, WGL. From the following formats in my research I worked mainly on circuit netlist and the format is Verilog.

\subsection{Challenges Faced Using Tetramax for RE}

- How to apply multi-input patterns at target gate.

- ATPG can sensitize a fault at one input (other input is non-controllable).

- How to propagate desired output value to PO.

- ATPG only propagates fault output.

- How to generate test pattern with incomplete netlist.

- ATPG need to know all gate types. 
All the challenges are addressed in the next chapter and proposed a methodology to RE the gate level netlist using ATPG tool. 


\section{CHAPTER 3}

\section{REVERSE ENGINEERING METHODLOGY USING ATPG TOOLS}

The methodologies to decamouflage involves gate-level and netlist-level test pattern generation. We also formulate the RE analysis based on the above analysis.

\subsection{Gate Level Input Pattern Identification}

This step generates the desired patterns to determine the logic implemented by the camouflaged gate. This is achieved by applying well-defined input test vectors and analyzing the

output response. The challenges associated with this step include: (i) minimizing the number of gate level input patterns required to identify the camouflaged logic; and, (ii) determining a methodology to exploit 'stuck-at' fault model of ATPG to apply the desired pattern at a target gate. These challenges are addressed below.

\subsubsection{Minimizing the Number of Gate Level Input Patterns}

To understand this challenge, we take the example of a 2 input gate with 4 input combinations. By analyzing the output generated for all these input combinations, the functionality of the gate can be determined. However, the challenge is to determine the logic implemented with minimum number of test patterns. To analyze this, we first populate a truth table with the 6 logic gates and their corresponding outputs for all the input combinations (Table-3.1). For example, in order to decipher a 'NAND' gate we first apply 00 pattern; a logic ' 1 ' output can correspond to NAND, NOR or XNOR logic gates. The next input combination chosen will be unique to that of 
Table 3.1 Test Patterns for Different Logic Gates

\begin{tabular}{|c|c|c|c|c|c|c|}
\hline Inputs & AND & OR & NAND & NOR & XOR & XNOR \\
\hline 00 & 0 & 0 & 1 & 1 & 0 & 1 \\
\hline 01 & 0 & 1 & 1 & 0 & 1 & 0 \\
\hline 10 & 0 & 1 & 1 & 0 & 1 & 0 \\
\hline 11 & 1 & 1 & 0 & 0 & 0 & 1 \\
\hline
\end{tabular}

the NAND gate (Table 3.2). By applying either a 01 or 10 pattern, only the NAND gate will output a logic 1 , while the other two gates will output a logic 0 . Therefore, we eliminate the need to extensively test the rest of the combinations to RE the functionality of a particular gate. Table 3.2 shows that a camouflaged gate that can assume 6 functionalities, can be fully reverse engineered using three test patterns ('00', ' 10 ' and ' 11 '). This in turn will reduce the time and number of patterns used to find the functionality of the camouflaged gate.

Table 3.2 Patterns Required to RE each Logic Function of Camouflaged Gate.

\begin{tabular}{|c|l|l|l|l|l|}
\hline AND & \multicolumn{1}{|c|}{ OR } & \multicolumn{1}{|c|}{ NAND } & NOR & XOR & XNOR \\
\hline$(00,10)$ & $(10,11)$ & $(00,10)$ & $(00,11)$ & $(00,11)$ & $(10,11)$ \\
\hline
\end{tabular}

Gate-level test pattern identification involves exhaustive search. To generalize this, we consider m-input gate that has $\mathrm{N}(=2 \mathrm{~m})$ possible patterns. Assuming the gate in isolation with $\mathrm{n}$ possible functionalities (e.g., NAND, NOR, AND, OR etc.) the table size will be nxN. In the worst case the optimal choice of $\mathrm{k}$ input pattern to $\mathrm{RE}$ each gate functionality will require searching through $\mathrm{N}$ patterns. The corresponding complexity will be $\sum_{k=1}^{N}{ }^{N} C_{k}$ which is $\sim \mathrm{O}\left(2^{\mathrm{N}}\right)$. For $\mathrm{n}$ functionalities the complexity is $\sim \mathrm{O}\left(\mathrm{nx} 2^{\mathrm{N}}\right)$. 


\subsubsection{Methodologies to Exploit Stuck at Fault Model}

Stuck-at fault as the name suggests is the event where an input of the gate is "stuck-at" a certain value (either 1 or 0 ). This is a well-established fault model for VLSI test [23]. This model can be exploited to apply the desired input pattern (as obtained from above analysis) at the target gate. For example, if a ' 00 ' pattern is required at the inputs of camouflaged gate then the adversary can apply stuck-at 1 fault at both inputs and generate the corresponding netlist-level test pattern.

In this context, it is worth mentioning that the conventional ATPG test pattern (for VLSI test) is determined in a way which not only sensitizes the fault but also propagates the fault effect to the primary output. In the proposed application of ATPG in RE, the output of a camouflaged gate is not known in advance which makes the fault propagation challenging. We resolve this issue by breaking the test pattern generation in two stages- first we find a pattern to sensitize the faults at a target gate, and, second, we find two patterns to propagate both ' 0 ' and ' 1 ' outputs of the gate to the PO. Finally, we merge the patterns to create two test patterns that can sensitize the faults and propagate both ' 0 ' and ' 1 ' output of the target gate.

\subsection{Netlist Level Test Pattern Generation Using ATPG}

Generation of netlist-level test pattern requires following steps: (i) dummy gate insertion in place of camouflaged gate to enable application of two faults at primary inputs; (ii) generation of test pattern for fault sensitization and propagation; and, (iii) stitching of test patterns. These are described below

\subsubsection{Dummy Gate Insertion}

In order to generate the netlist-level test patterns, we first replace the target camouflaged gate with an appropriate dummy gate. The choice of dummy gate depends on the gate-level pattern that needs to be applied at the inputs of camouflaged gate as well as the output. The unique feature 
of ATPG tools such as Tetramax is that when a stuck-at fault is placed at one of the input, the other input is automatically set to non-controlling value (depending on the gate type) for fault sensitization. For example, when a stuck-at 0 fault is placed at one input of NAND gate then the one input receives a logic ' 1 ' whereas other input is automatically set to logic ' 1 '. Therefore, by choosing to replace the camouflaged gate with NAND gate '11' pattern could be applied. We exploit this feature to apply two inputs at a gate with the stuck-at fault model of ATPG tools which allow insertion of single fault at a time. Similarly, a dummy AND or XNOR gate can enable fault sensitization and propagation if pattern ' 11 ' needs to be applied at the inputs. Therefore, the camouflaged gate should be replaced with XNOR/AND for test pattern generation. XOR dummy gate can enable generation of 10/01 pattern. TABLE-3.3 shows the desired gate-level patterns and corresponding dummy gate types that can be used to generate patterns using ATPG. Note that multiple choices of a dummy gate are possible for a desired gate-level test pattern.

Table 3.3 Gate Level Patterns and Corresponding Dummy Gate Types

\begin{tabular}{|c|c|c|}
\hline $\mathrm{I}_{1} \mathrm{I}_{2}$ & Dummy gate types & Fault Injection $\left(\mathrm{I}_{1}, \mathrm{I}_{2}\right)$ \\
\hline 00 & AND,XOR,OR & $(\mathrm{sa} 1, \mathrm{x})$ \\
\hline & NAND & $(\mathrm{x}, \mathrm{sa} 0) /(\mathrm{sa} 0, \mathrm{x})$ \\
& NOR & $(\mathrm{x}, \mathrm{sa} 1) /(\mathrm{sa} 1, \mathrm{x})$ \\
$01 / 10$ & AND & $(\mathrm{sa} 1, \mathrm{x}) /(\mathrm{x}, \mathrm{sa} 1)$ \\
& XOR & $(\mathrm{x}, \mathrm{sa} 0) /(\mathrm{sa} 0, \mathrm{x})$ \\
& OR & $(\mathrm{x}, \mathrm{sa} 0) /(\mathrm{sa} 0, \mathrm{x})$ \\
& XNOR & $(\mathrm{sa} 1, \mathrm{x}) /(\mathrm{x}, \mathrm{sa} 1)$ \\
\hline 11 & AND,XNOR,OR & $(\mathrm{sa} 0, \mathrm{x})$ \\
\hline
\end{tabular}

\subsubsection{Fault Sensitization and Propagation}

Stuck-at fault and dummy gate are introduced in the netlist at the camouflaged gate to obtain netlist-level test pattern. For example, stuck-at 1 and dummy AND gate in the netlist is employed to ensure pattern '01' is applied in Fig 3.1. The patterns generated in such manner will 
ensure that the desired gate-level pattern is applied to the camouflaged gate. The next objective is to propagate the output of camouflaged gate to a PO. Note that the pattern needed to propagate the fault could be orthogonal to the pattern needed to sensitize the faults. Therefore, the two patterns could be stitched together to generate a pattern that can apply the desired input at the camouflaged gate and propagate the output to PO. In example shown in Fig 3.1 the inputs required for the fault sensitization and propagation are $\{\mathrm{N} 3, \mathrm{~N} 6, \mathrm{~N} 7\}$ and $\{\mathrm{N} 2, \mathrm{~N} 6, \mathrm{~N} 7\}$ respectively.

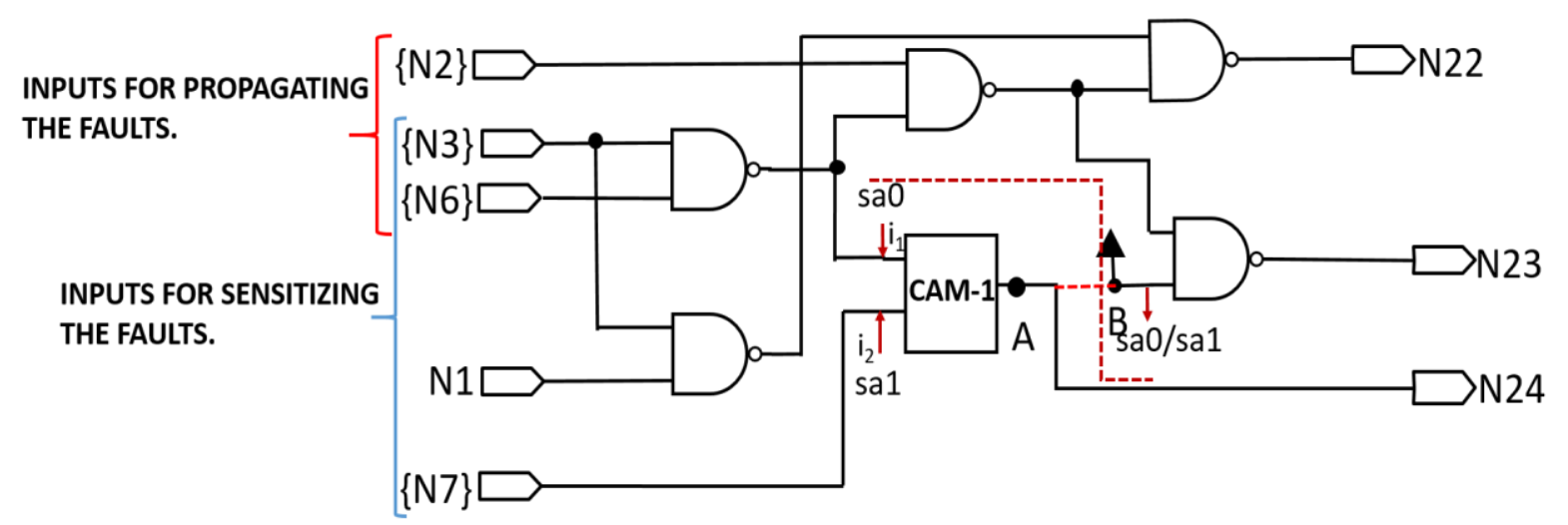

Figure 3.1 De-camouflaging a Gate in C17 using Split and Stitch Method.

\subsubsection{Split and Stitch Methodology}

In order to prepare the netlist for test generation we first split the output of the camouflaged gate (node A) and make it a PO. The fan-out of camouflaged gate (node B) is grounded for the purpose of test generation (Fig 3.1). Next stuck-at fault and dummy gate is introduced in the netlist and test pattern is generated. After storing the resulting pattern (P1) we connect nodes A\&B back and introduce a stuck-at 1 and stuck-at 0 fault respectively at that node, and store the generated patterns $\mathrm{P} 2$ and P3. Note that P1 applies the desired pattern on camouflaged gate and P2/P3 propagates the output ( 0 or 1 ) to PO. Furthermore, the PIs used to sensitize and propagate are orthogonal. Therefore, stitching (P1, P2) and (P1, P3) will provide two new patterns that can attain 
the desired objective. Similar step can be followed to obtain the test patterns to apply all desired inputs on the camouflaged gate and observe its response.

\subsection{Reverse Engineering Effort}

The RE effort is a function of time required to, (i) identify gate-level test pattern ( $\left.\mathrm{T}_{\text {gate_level }}\right)$; (ii) determine and insert dummy gates $\left(\mathrm{T}_{\text {dummy }}\right)$; (iii) inject stuck-at fault at gate input $\left(\mathrm{T}_{\text {fault_injection }}\right)$; (iv) find the pattern by the ATPG tool $\left(\mathrm{T}_{\mathrm{ATPG}}\right)$; (v) propagate the fault effect to the $\mathrm{PO}$ ( $\left.\mathrm{T}_{\text {propagation}}\right)$; and, (vi) find the common pattern to sensitize and propagate the fault ( $\mathrm{T}_{\text {intersection }}$ ). It must be noted that the RE effort will depend on the number of camouflaged gates in the circuit, number of possible functionalities exhibited by the camouflaged gate, number of inputs to the dummy gate and time taken by the ATPG tool. The effort is given by,

RE effort $=\mathrm{T}_{\text {gate_level }}+\mathrm{T}_{\text {dummy }}+\left[\left(\mathrm{T}_{\text {fault injection }}+\mathrm{T}_{\mathrm{ATPG}}\right)+\left(\mathrm{T}_{\text {fault injection }}+\mathrm{T}_{\mathrm{ATPG}}\right)\right]+\left\{2 *\left[\mathrm{~T}_{\text {propagation }}+\right.\right.$ $\left.\left.\mathrm{T}_{\mathrm{ATPG}}\right]+2 * \mathrm{~T}_{\text {intersection }}\right\}$

Note that in the above equation a factor of 2 is included to account for logic ' 0 ' and ' 1 ' propagation from gate output. $\mathrm{T}_{\mathrm{ATPG}}$ depends on the complexity of algorithm used in ATPG tool and the machine specification. 


\section{CHAPTER 4}

\section{CAMOUFLAGED GATES ${ }^{2}$}

In this chapter we discuss about the camouflaged gate and multiplexer that we used in the thesis to calculate the Reverse Engineering effect.

\subsection{Threshold Voltage Defined Switch Design}

Fig 2.1 shows the threshold voltage defined switch which is either a PMOS or an NMOS transistor. The threshold voltage of the switch (transistor) is adjusted such that it is either ON or OFF. Low threshold voltage (LVT) turns the switch ON as it offers low resistance and high threshold voltage (HVT) turns the switch OFF because of the high resistance offered. The deviation of threshold voltage $(\Delta \mathrm{VT})$ from the normal threshold voltage (NVT) which gives an optimum value of delay and leakage power is selected to operate the switch in the camouflage gate. The gate voltage of the switch is selected such that Vgs (Gate to source voltage) of the transistor is greater than LVT making the switch ON and lower than HVT making the switch OFF. Thus, the switch turns ON/OFF based on the threshold voltage (VT) asserted on it and the applied chosen gate voltage. For N-switch, high HVT and low gate bias voltage is good for leakage while low LVT, high gate bias voltage is good for performance and vice-versa for PMOS switch.

\footnotetext{
${ }^{2}$ The following chapters are previously published in [23]. Permissions are included in Appendix A.
} 


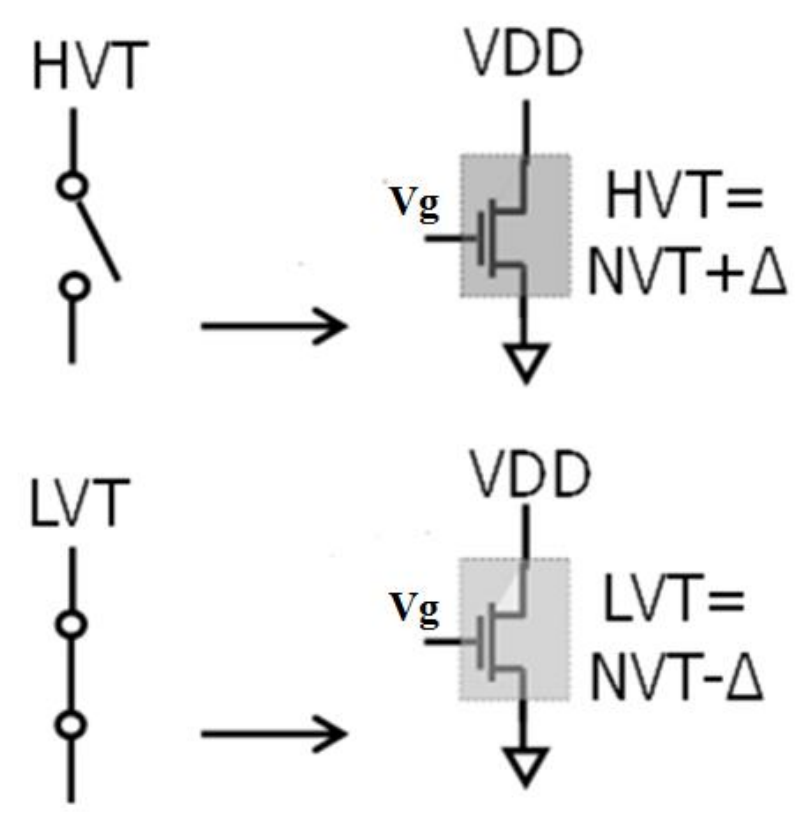

Figure 4.1 PMOS/NMOS Switch ON/OFF Demonstration with Gate Voltage Vg

\subsection{Proposed Camouflage Gate}

The threshold voltage defined switches are used in conjunction with NVT transistors to camouflage a gate. In this thesis, we propose a logic gate using VT defined switches that can perform AND, OR, NAND, NOR, XOR and XNOR operations. Thus, the gate can be used to camouflage any other logic gate in the circuit [24].

Fig 4.2 shows the schematic of camouflaged gate that exhibits 6-functionalitites (AND, OR, NAND, NOR, XOR and XNOR). The switches that have to be ON (asserted to LVT) to realize different functionalities is shown in Table 4.1. For example, by asserting LVT on switches S2 and S7 and HVT on all other switches, a parallel connection of PMOS transistors and a series connection of NMOS transistors can be obtained and a AND logic can be realized. 
Note that the performance and area of the proposed camouflaged gate is strongly correlated to the resistance of ON and OFF switches in the path. Considering the widths of all the switches, area of the proposed camouflaged design is calculated to be $2.64 \mu \mathrm{m}^{2}$.

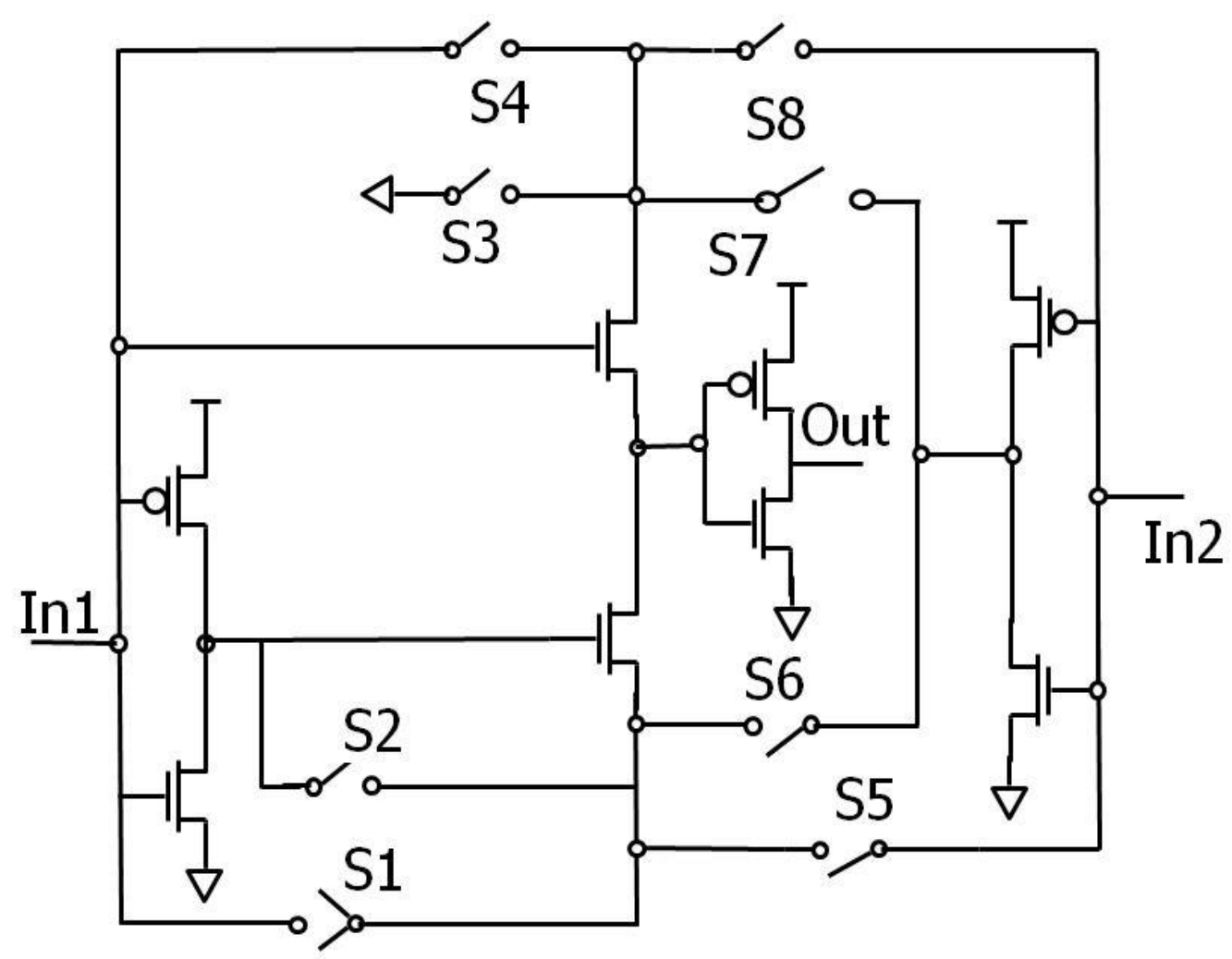

Figure 4.2 Camouflaged Gate with VT Defined Switches 
Table 4.1 Different Logic Implementation of Camouflage Gate.

\begin{tabular}{|c|c|}
\hline Logic & ON (LVT) switches \\
\hline AND & S2, S7 \\
\hline OR & S3, S6 \\
\hline NAND & S1, S8 \\
\hline NOR & S4, S5 \\
\hline XOR & S6, S8 \\
\hline XNOR & S5, S7 \\
\hline
\end{tabular}

\subsection{Threshold Voltage Defined Multiplexer}

$\mathrm{N}-1$ multiplexer design will have N CMOS switches with one switch is LVT and N-1 are HVT switches. Fig 4.6 shows a 2-1 multiplexer. A good $V_{T}$ defined switch should offer high ON current and low OFF current. The gate voltage, HVT, LVT values and transistor sizes are tuned to maximize the $\mathrm{I}_{\mathrm{ON}} / \mathrm{I}_{\mathrm{OFF}}$ ratio. For $\mathrm{N}$-switch, higher HVT values and lower gate voltage is good for leakage whereas lower LVT and higher gate voltage is good for performance and vice-versa is true for P-switch. The switch is characterized by LVT and HVT values and switch bias voltage that results in high $\mathrm{I}_{\mathrm{ON}} / \mathrm{I}_{\mathrm{OFF}}$ ratio and optimized delay. 

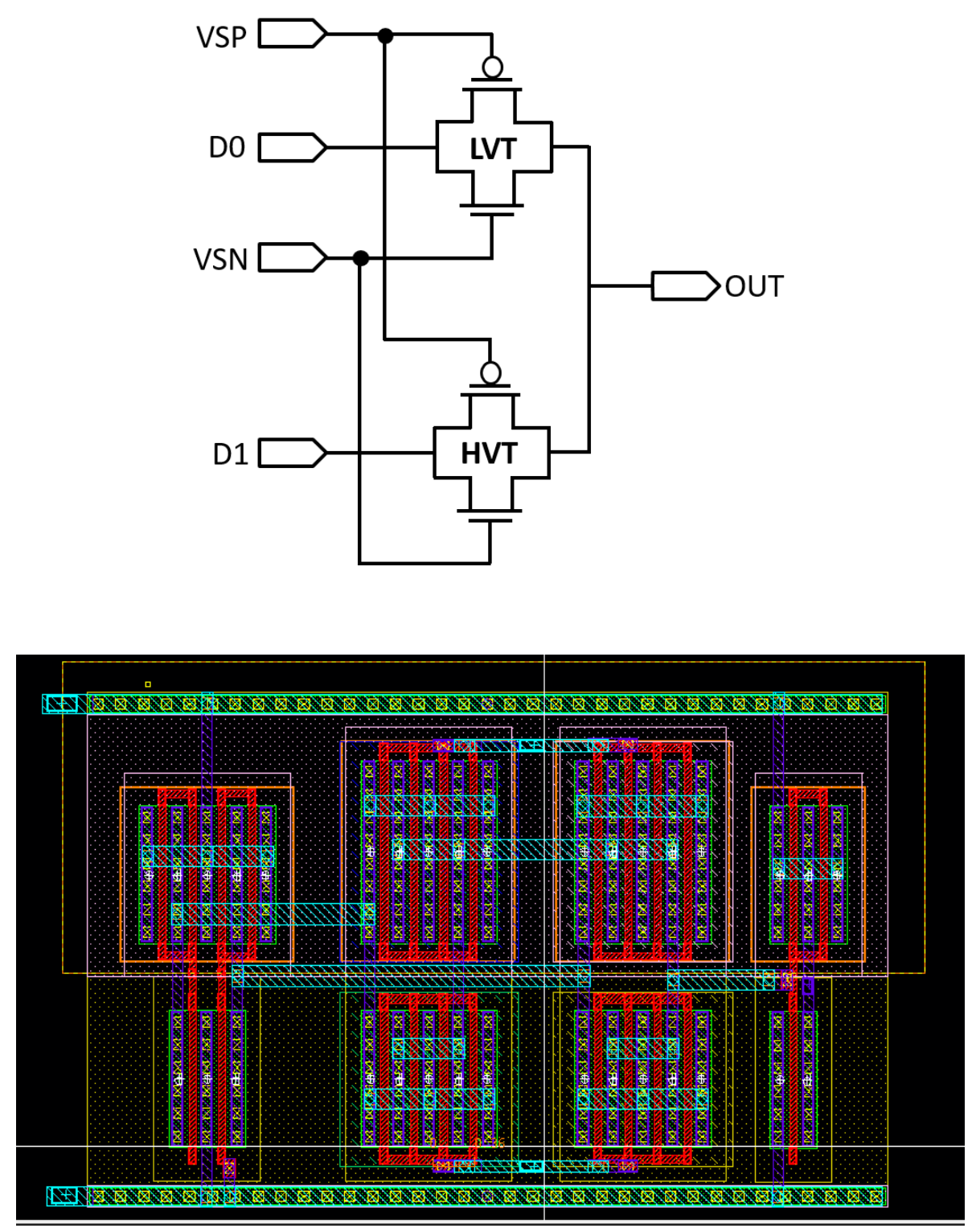

Figure 4.3 Schematic and Layout of Threshold Defined 2-1 Multiplexer. 


\section{CHAPTER 5}

\section{MULTIPLE CAMOUFLAGED GATES}

It is not sufficient to camouflage a single gate in the entire netlist to protect against RE. It is therefore imperative to camouflage multiple gates. Multiple camouflaged gates can be realized under one of the following circumstances: (i) the gates are completely isolated from one another, meaning they don't have any common inputs and the fault of one gate doesn't affect the other gates (i.e., no edge between the two camouflaged gates); and, (ii) the gates are dependent on each other (i.e. a common edge is present between the two camouflaged gates). In this section, we address both these circumstances in terms of RE effort.

\subsection{RE of Two Isolated Camouflaged Gates}

This is the easiest case as shown in Fig 5.1 where the same de-camouflaging approach can

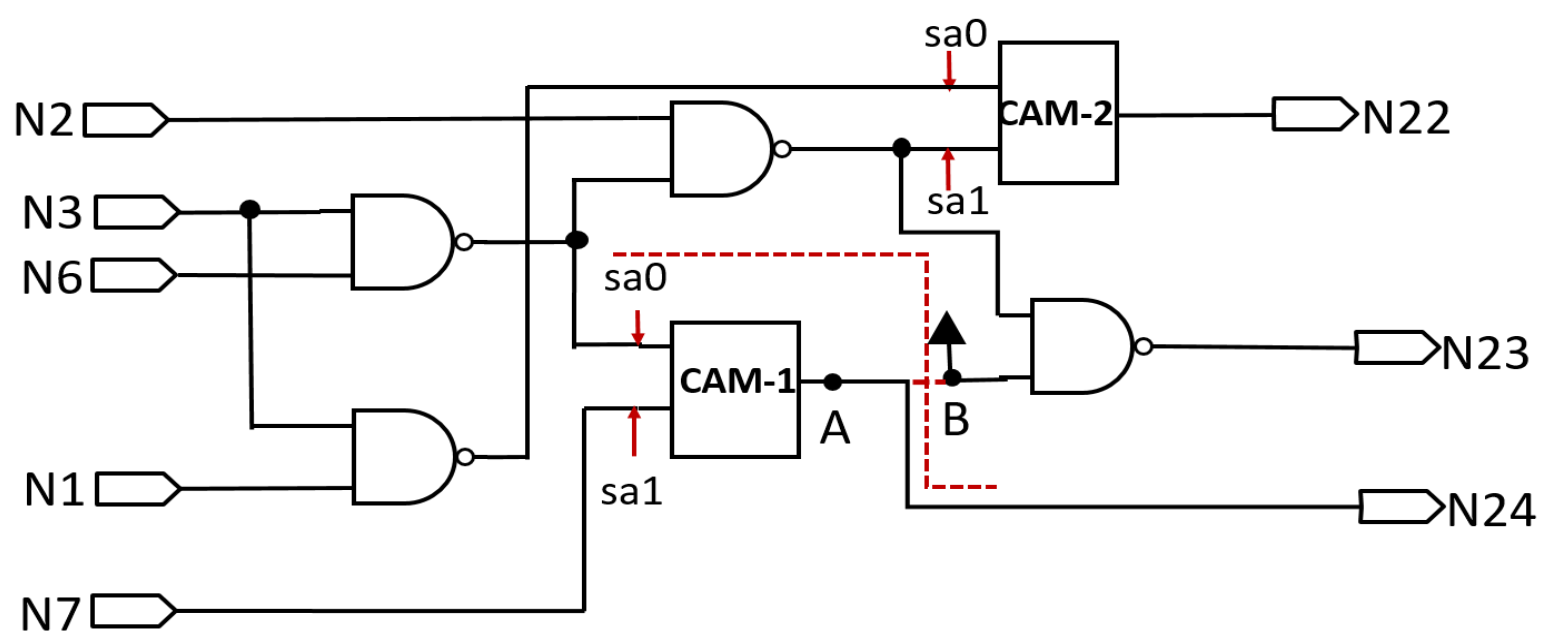

Figure 5.1 C17 Benchmark with Two Independent Camouflaged Gates. 
be followed as single camouflaged gate. First a gate is picked and the test patterns are found. By employing the patterns on the chip the gate could be de-camouflaged. The netlist is updated and the same process is repeated till all gates are de-camouflaged.

\subsection{RE of Two Series Connected Camouflaged Gates}

In this case we combine both camouflaged gates and introduce a three input gate (with one or multiple outputs depending on fanouts of both gates) in place of two gates as shown in Fig 5.2. Next we can introduce the faults and generate the patterns similar to the single camouflaged gate. However, this case will have 36 possible combinations of possible logic (assuming each gate exhibits 6 functionalities) and 8 possible input patterns.

Similar to discussion in Chapter II, unique gate-level patterns need to be identified to decamouflage all possible 36 cases using less than 8 patterns. Once the patterns are identified the choice of dummy gates are made for netlist-level test pattern generation. Note that the RE effort will increase due to increased complexity of test pattern generation.

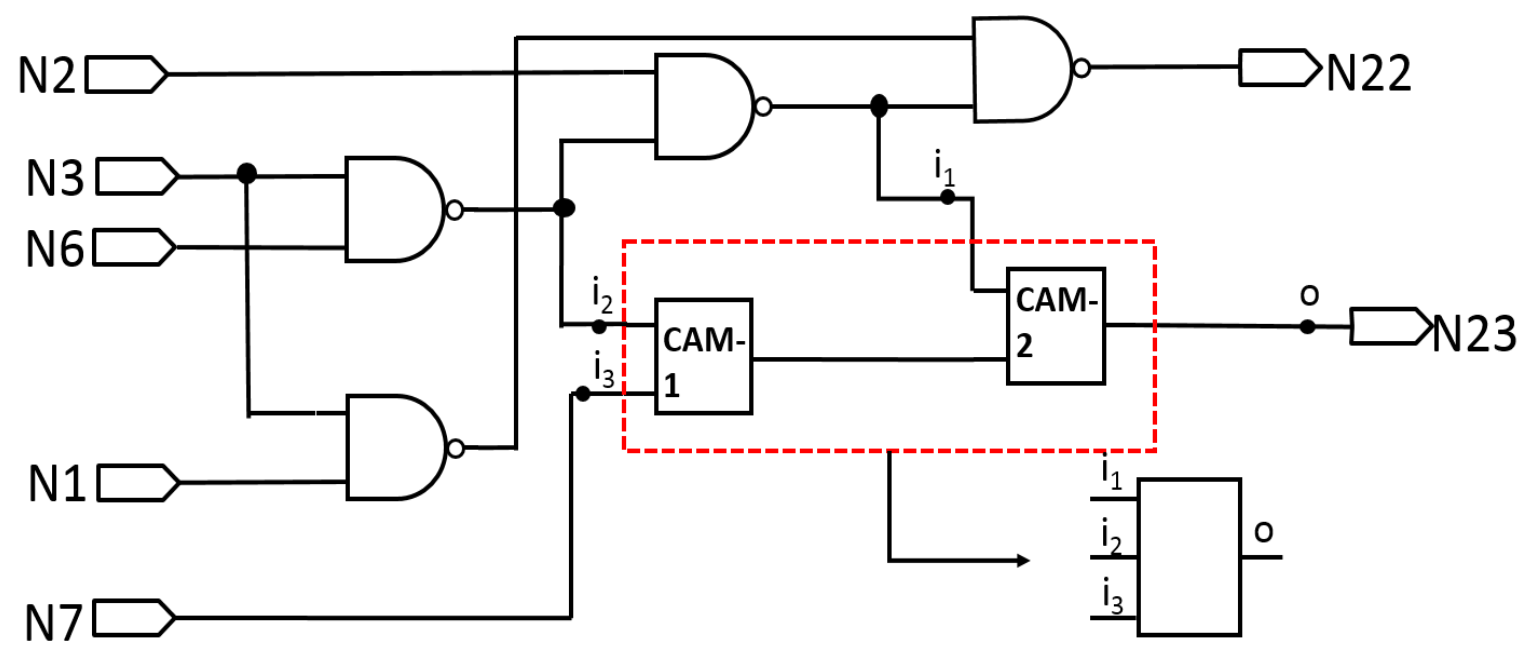

Figure 5.2 C17 Benchmark with Series Connected Camouflaged Gates. 


\subsection{RE of Two Camouflaged Gates with a Known Gate in Between}

In this scenario a known gate is sandwiched between two camouflaged gates. We have two possibilities in this case: (i) a gate (Fig 5.3(a)) with more than one fanout; and, (ii) a gate with only one fanout (Fig 5.3(b)). When the known gate has more than one fanout, the fault effect could be propagated to a PO through alternative paths. Therefore, this situation is same as two independent gates and the split-and-stitch method can be directly applied on each individual camouflaged gate. With a single fanout (Fig 5.3(b)) however, we need to combine the three gates and replace them with a four input gate. In this example, we get a possible 36 logical combinations, with a total of 16 possible input combinations.

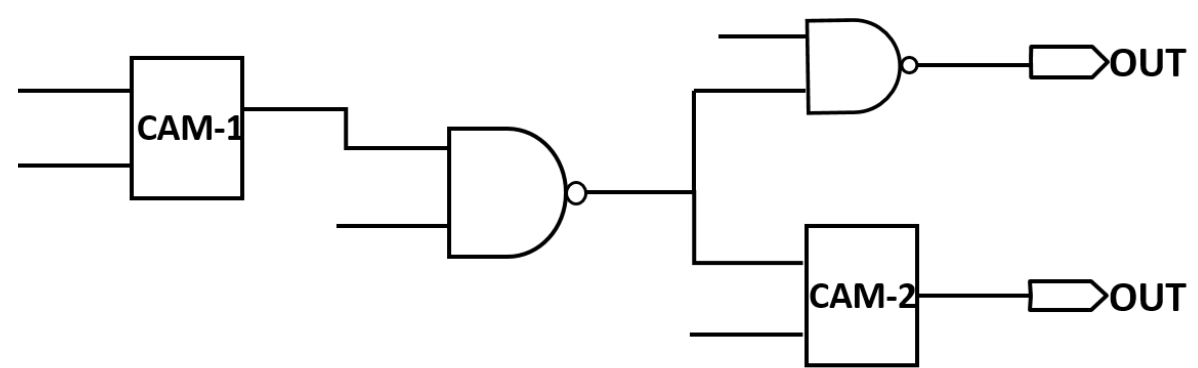

(a)

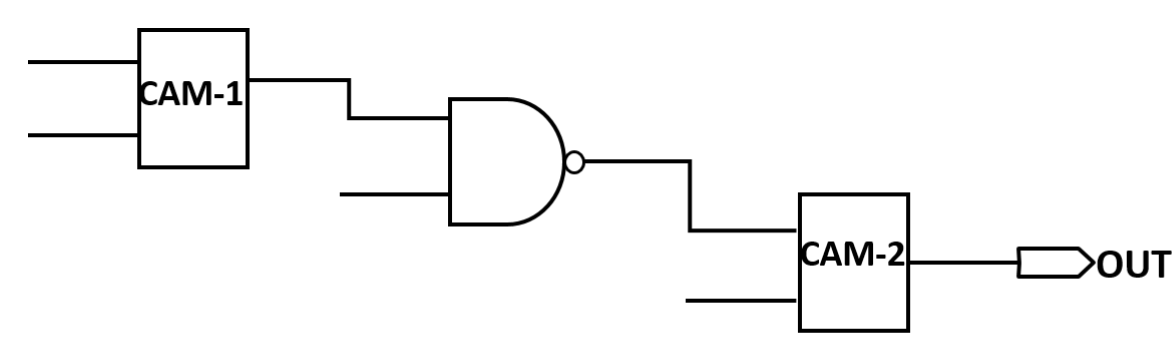

(b)

Figure 5.3 A Known Gate in Between Two Camouflaged Gates. (a) with More Than One Fanout; and, (b) with One Fanout. 


\subsection{Three Series Connected Camouflaged Gates}

In this case as we replace the three camouflaged gates with a four input gate (similar to Section 5.3) as shown in Fig 5.4. The RE complexity proportionally increases as each gate now has 6 possible combinations giving rise to 216 different possible logical combinations with 16 possible input patterns. It is observed that a minimum of 6 patterns are required to de-camouflage a single 3-gate combination design. It must also be noted that some of the possible gate combinations are functionally equivalent (i.e. XOR-XOR-XOR gate is functionally equivalent to XNOR-XNOR-XNOR gate).

Therefore, once the RE boils down to functionally equivalent logic, the gates are assumed to be successfully de-camouflaged. Fig 5.5 shows the number of gate-level patterns required to identify the functionalities of single, two series-connected and three series-connected camouflaged gates. It is observed that the RE effort grows rapidly as more camouflaged gates are daisy chained.

\subsection{Camouflaging Using Threshold Defined Multiplexer}

In this section we present the threshold defined multiplexer and key design requirements and obfuscation methodology.

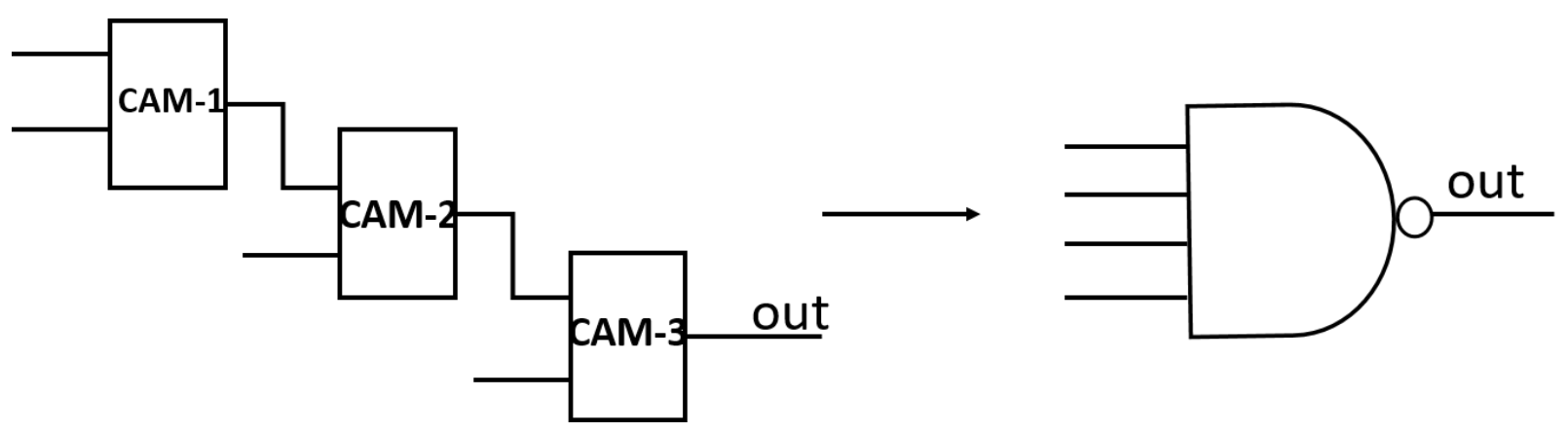

Figure 5.4 Three Series Connected Camouflaged Gates 


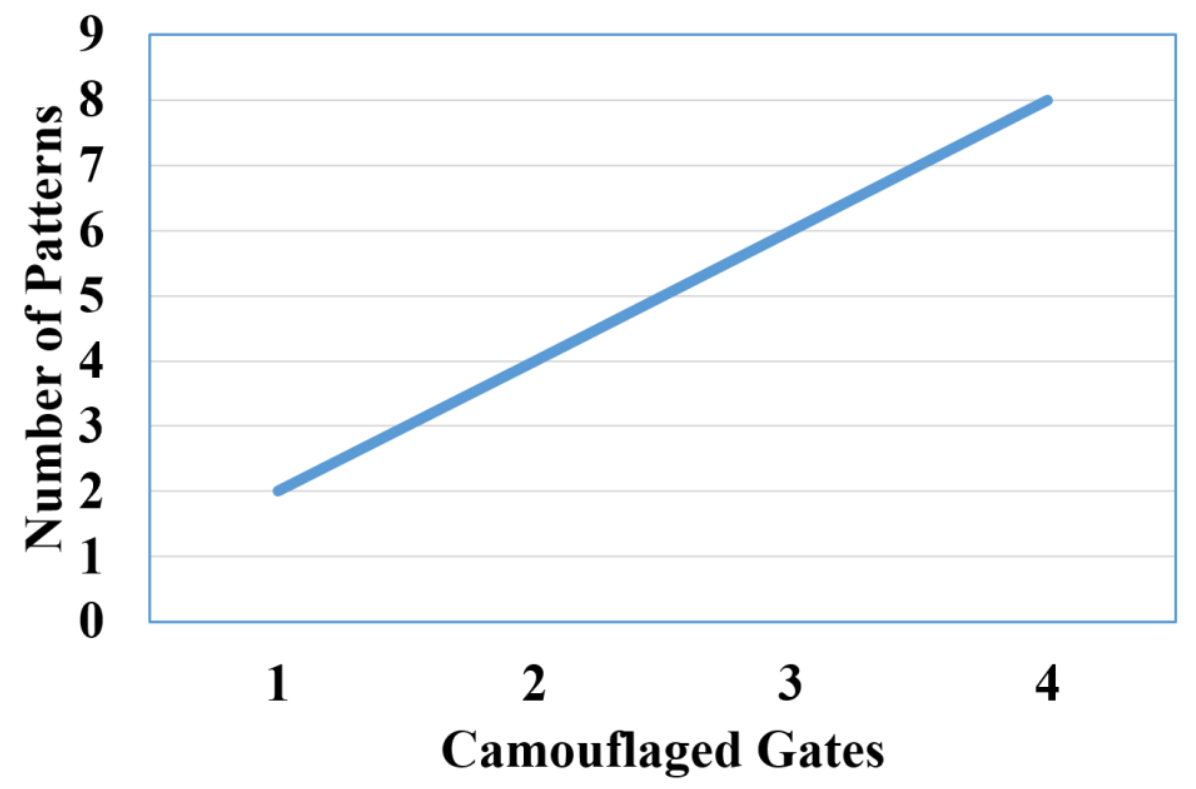

Figure 5.5 Number of Input Patterns vs Number of Series Connected Camouflaged Gates

\subsubsection{Obfuscation Methodology}

In the previous sections we saw the camouflaging using a gate now we will camouflage the netlist using the threshold defined multiplexer. Using the multiplexer, we will camouflage the interconnects as shown in Fig 5.6. The adversary will be confused trying to find which is the original path. For selecting the nets, we again use same metrics that is controllability and observability which are given in detail in the following chapter. In addition to these metrics the one condition that is to be satisfied is, net should have a fanout greater than or equal to 2 .

\subsubsection{RE of Multiplexer}

RE effort in this case will be the time taken to set the inputs and time taken to propogate the output. $\mathrm{RE}=\mathrm{T}_{\text {setting }}+\mathrm{T}_{\text {propogation. }}$. Each input has two possibilities that is either 1 or 0 as we are using 2-1 multiplexer we will have two patterns 10 and 01 at the input and we need to propogate the output in both cases. 


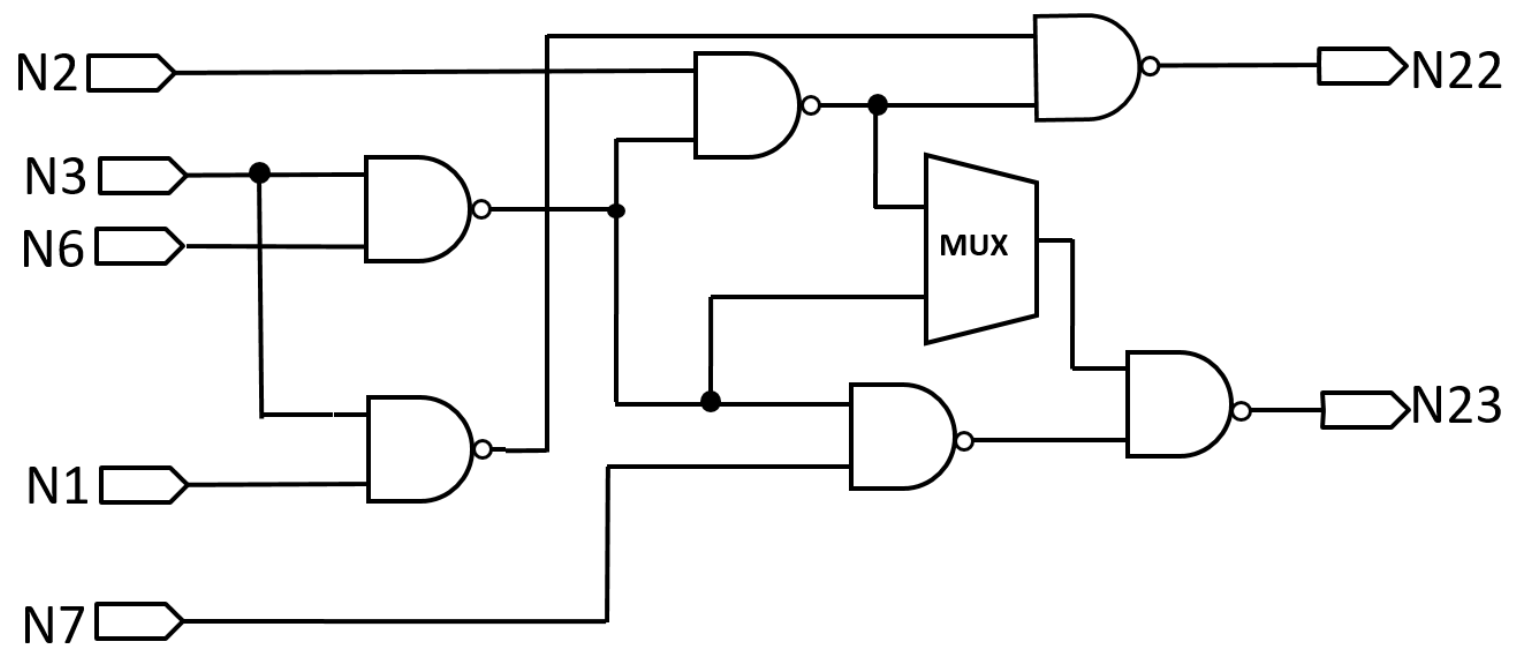

Figure 5.6 C17 Benchmark after Replacing the Selected Nets by Multiplexers. 


\section{CHAPTER 6}

\section{CAMOUFLAGING TECHNIQUES}

In this chapter we present techniques to increase the RE effort using metrics such as controllability, observability and HD sensitivity.

\subsection{Controllability and Observability-Based Technique}

We calculate the controllability and observability [12] of each gate in the netlist. The gates connected to the Primary Input (PIs) are hard to observe but easy to control. However the gates deeper in the logic becomes difficult to observe but hard to control. The gates that are both hardto-control (HTC) and hard-to-observe (HTO) are ideal choices for camouflaging. However,

Table 6.1 Controllability and Observability Calculations for Two Input Gates.

\begin{tabular}{|c|c|c|}
\hline LOGIC & OBSERVABILITY & CONTROLLABILITY \\
\hline AND & $\begin{array}{l}\operatorname{CO}(a)=C O(z)+\operatorname{CC} 1(b)+1 \\
\operatorname{CO}(b)=C O(z)+\operatorname{CC} 1(a)+1\end{array}$ & $\begin{array}{l}\operatorname{CCO}(z)=\min (\operatorname{CCO}(a), \operatorname{CCO}(b))+1 \\
\operatorname{CC1}(z)=\operatorname{CC1}(a)+\operatorname{CC1}(b)+1\end{array}$ \\
\hline OR & $\begin{array}{l}\operatorname{CO}(a)=C O(z)+\operatorname{CCO}(b)+1 \\
\operatorname{CO}(b)=\operatorname{CO}(z)+\operatorname{CCO}(a)+1\end{array}$ & $\begin{array}{l}\operatorname{CCO}(z)=\operatorname{CCO}(a)+\operatorname{CCO}(b)+1 \\
\operatorname{CC1}(z)=\min (\operatorname{CC1}(a), \operatorname{CC1}(b))+1\end{array}$ \\
\hline NAND & $\begin{array}{l}\mathrm{CO}(a)=C O(z)+\operatorname{CC} 1(b)+1 \\
\operatorname{CO}(b)=C O(z)+\operatorname{CC1}(a)+1\end{array}$ & $\begin{array}{l}\operatorname{cCO}(z)=\operatorname{CC1}(a)+\operatorname{CC1}(b)+1 \\
\operatorname{cC1}(z)=\min (\operatorname{CCO}(a), \operatorname{CC1}(b))+1\end{array}$ \\
\hline NOR & $\begin{array}{l}C O(a)=C O(z)+\min (C C O(b), C C 1(b))+1 \\
\operatorname{CO}(b)=C O(z)+\min (C C O(a), C C 1(a))+1\end{array}$ & $\begin{array}{l}\operatorname{CCO}(z)=\min (C C O(a)+C C O(b), C C 1(a)+C C 1(b))+1 \\
\operatorname{CC1}(z)=\min (C C 1(a)+C C O(b), C C O(a)+C C 1(b))+1\end{array}$ \\
\hline XOR & $\begin{array}{l}\mathrm{CO}(a)=\operatorname{CO}(z)+C C O(b)+1 \\
\operatorname{CO}(b)=C O(z)+C C O(a)+1\end{array}$ & $\begin{array}{l}\operatorname{CCO}(z)=\min (\operatorname{CC} 1(a), \operatorname{CC1}(b))+1 \\
\operatorname{CC1}(z)=\operatorname{CCO}(a)+\operatorname{CCO}(b)+1\end{array}$ \\
\hline XNOR & $\begin{array}{l}C O(a)=C O(z)+\min (C C O(b), C C 1(b))+1 \\
\operatorname{CO}(b)=C O(z)+\min (C C O(a), C C 1(a))+1\end{array}$ & $\begin{array}{l}C C O(z)=\min (C C 1(a)+C C O(b), C C O(a)+C C 1(b))+1 \\
\operatorname{CC1}(z)=\min (C C O(a)+C C O(b), C C 1(a)+C C 1(b))+1\end{array}$ \\
\hline $\begin{array}{l}\text { INVERT } \\
\text { ER }\end{array}$ & $\mathrm{CO}(\mathrm{a})=\mathrm{CO}(\mathrm{Z})+1$ & $\begin{array}{l}\operatorname{CCO}(z)=\operatorname{CC1}(a)+1 \\
\operatorname{CC1}(z)=\operatorname{CCO}(a)+1\end{array}$ \\
\hline
\end{tabular}




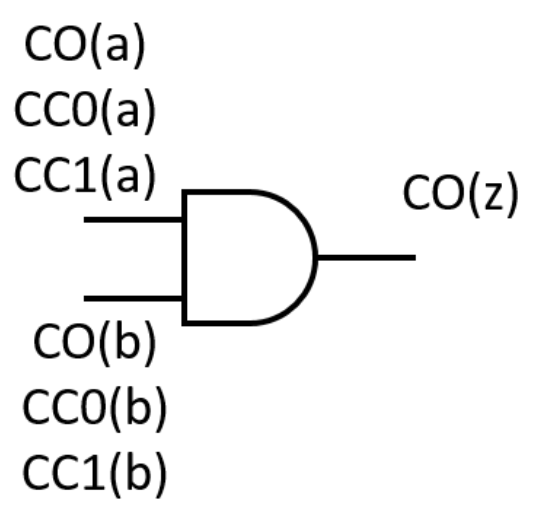

Figure 6.1 Controllability and Observability Formulae Implementation

finding such gates in the design is nearly impossible. Therefore, we add controllability (CC0 and $\mathrm{CC} 1)$ and observability $(\mathrm{O})$ values of the gate to determine an aggregate metric (HTCO) that is representative of its overall hardness for $\mathrm{RE}$ and is given by, $\mathrm{HTCO}=\mathrm{CC} 0+\mathrm{CC} 1+\mathrm{O}$.

A graph is created after reading the netlist where each node corresponds to a gate and the edge corresponds to their connectivity. The graph is traversed from PI to PO (PO to PI) and the controllability (observability) values of each node is computed. A list of gates is created in descending order of HTCO values and top gates are selected for camouflaging. As noted before the selected gates could either be independent of each other or could be a mixture of indepndent and dependent (series connected) gates. We evaluate the RE effort for both these conditions.

\subsection{Hamming Distance Sensitivity-Based Technique}

The Hamming Distance Sensitivity (HDS) is analytically computed by adding the differential change in POs by changing the response of a gate for a exhaustive set of test vectors. Theoretically it determines the ability of a gate to maximize the change in POs for a given test vector. It is notable that a gate which can be reached from maximum number of POs will have higher potential to affect the POs. Therefore such gates will have higher HDS. In this paper we use the reachability of the gate from POs as representative of HDS. This is achieved by traversing 
the graph (corresponding to a netlist) in depth-first manner and computing the number of POs reached from each gate.

HDS $\left(\right.$ Gate $\left._{i}\right)=$ Number of POs that gate can be reached from Gate ${ }_{i}$

A list of gates is created in descending order of HDS values and top gates are selected for camouflaging. In addition to HTCO and HDS, we also evaluate the efficiency of an aggregate metric called hard-to-RE (HTRE) that constitutes controllability, observability and HDS, and, is given by HTRE=HTCO +HDS. 


\section{CHAPTER 7}

\section{RESULTS AND ANALYSIS}

We evaluate proposed camouflaging [25] and RE technique using ISCAS85 benchmarks [26]. We implement the gate selection algorithm in C to calculate the HTCO [23], HDS and HTRE of each gate. Next, we select $5 \%, 10 \%$ and $15 \%$ of total number of gates for camouflaging. A random selection is also implemented for comparison. We evaluate the RE effort (using (1)) of independent, dependent (2-series connected) as well as mixture of independent and dependent gates. In RE effort estimation a clock frequency of $1 \mathrm{GHz}$ (1ns cycle time) is assumed for each combination of gate-level test pattern generation/application. The ATPG time is determined by executing it on linux 6.5 carbon with AMD operton processor (2GHz clock and 32GB RAM). Since camouflaging is associated with area, delay and power overhead, the objective is to maximize the RE effort with less percentage of camouflaging.

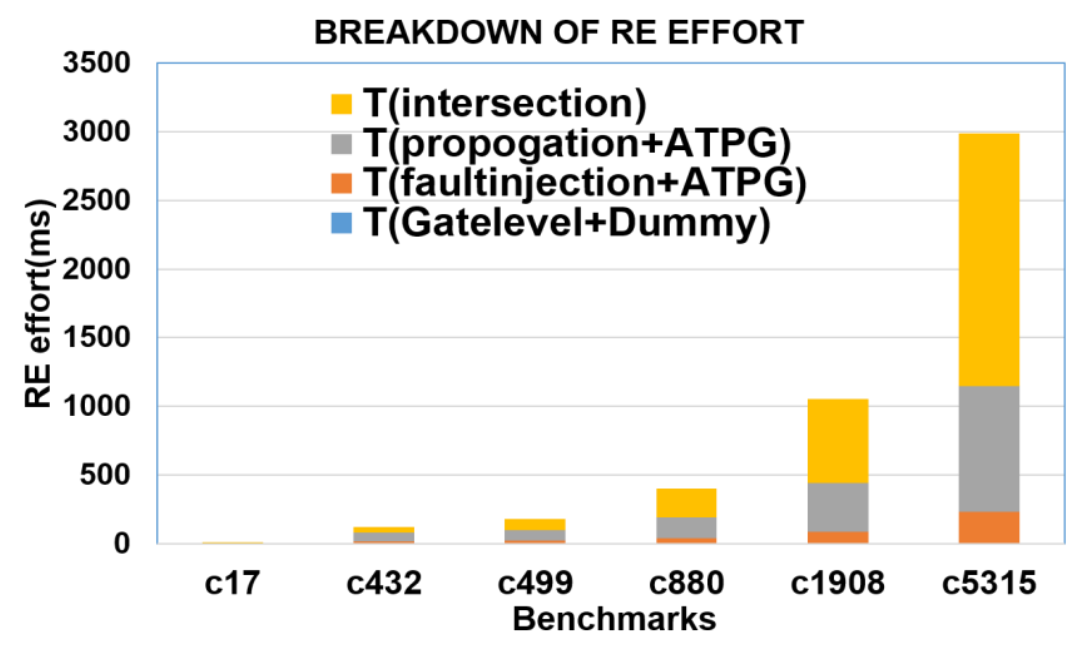

Figure 7.1 Breakdown of RE Effort for HTRE with 5\% Camouflaging 


\subsection{Camouflaged Gate RE Results}

Fig 7.1 shows an example of RE effort breakdown for HTRE metric with 5\% camouflaging. It can be observed that majority of RE effort is spent in finding the intersection of netlist-level test patterns and to find test pattern for propagation of gate output.

The RE effort for random gate selection, HTCO with independent gates (HTCO(indep)), HTCO with 2-series dependent gates $(\operatorname{HTCO}(2$-series) ) and $\mathrm{HTCO}$ with pure gate selection respectively are shown in Fig 7.2. HTCO(indep) is obtained by eliminating the dependent gates and picking the independent gates from list of gates that are sorted in decremental order of HTCO metric.
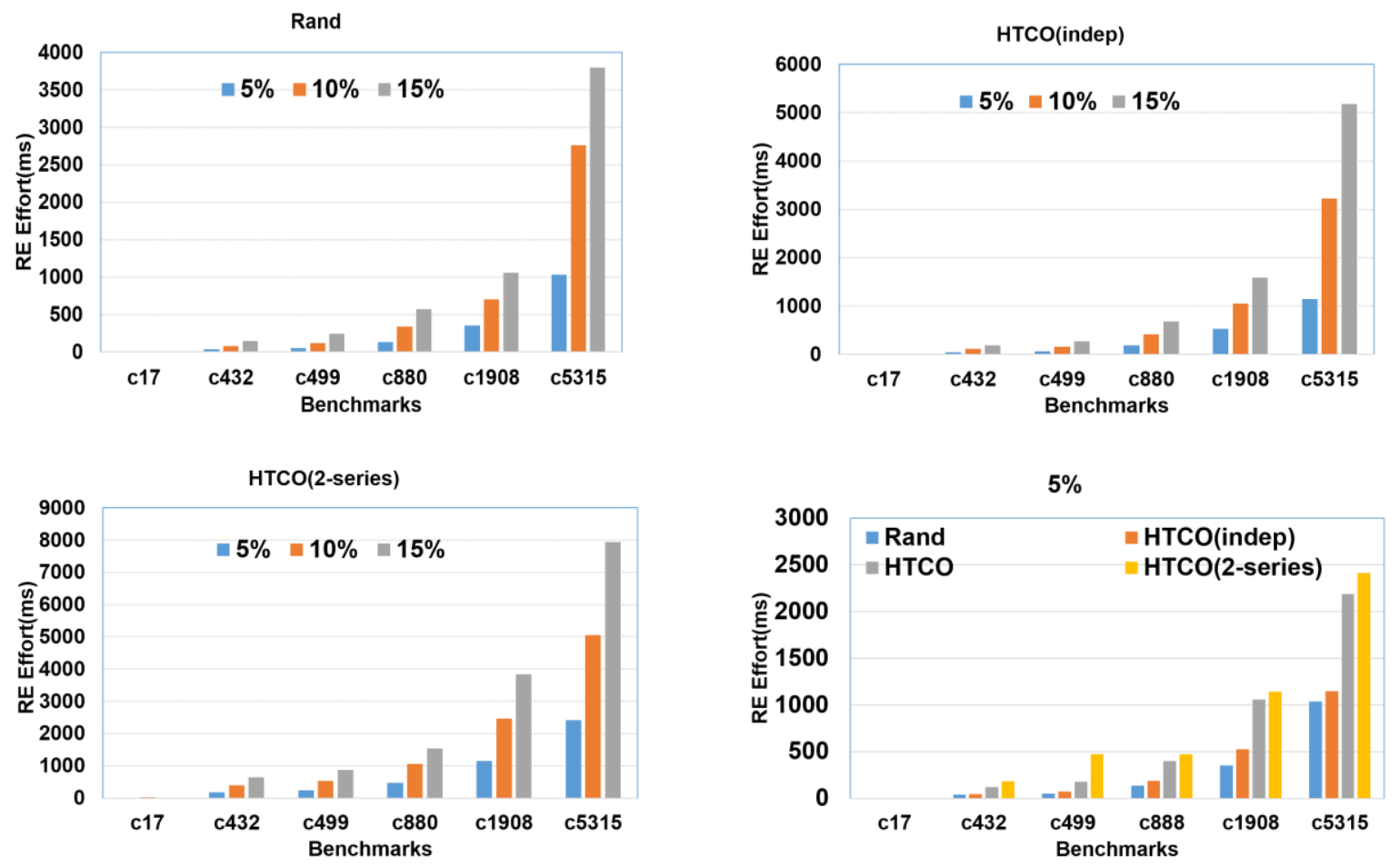

Figure 7.2 RE Effort for Different Benchmarks using HTCO. 
Similarly, HTCO (2-series) is obtained by eliminating the independent gates and more than 2-series connected gates.
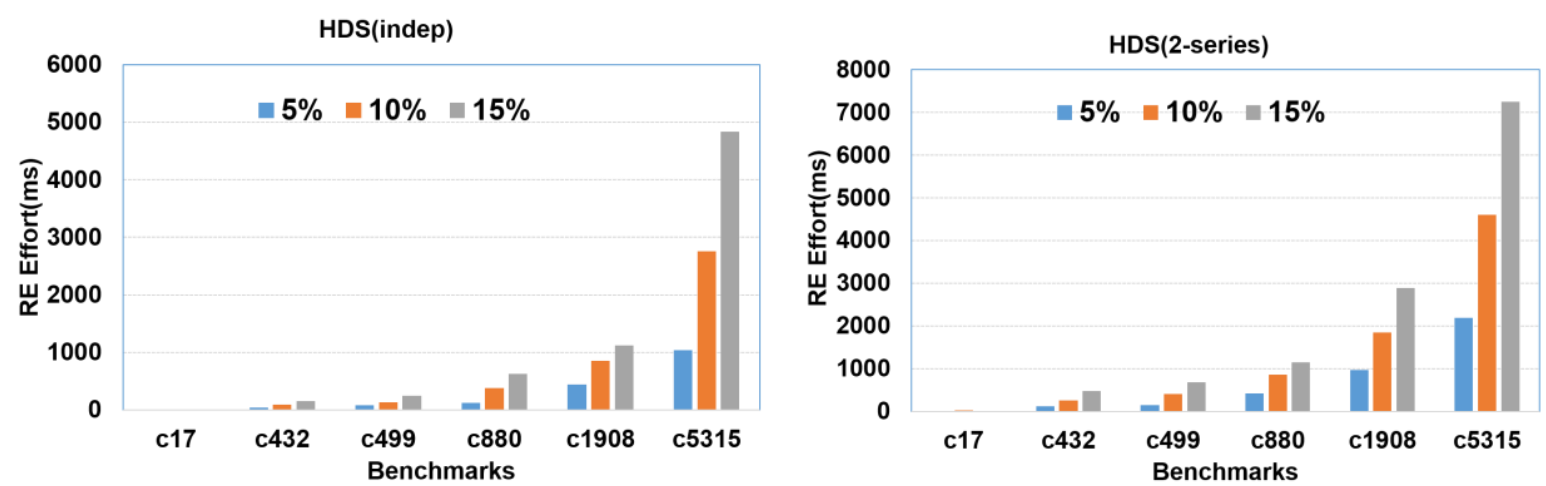

Figure 7.3 RE Effort for HDS with Independent and 2-Series Dependent Gates.

It can be noted that the RE effort increases significantly with when only series connected gates are picked for camouflaging instead of independent gates that are hard-to-control and hardto-observe. This conclusion is more evident in Fig 7.2 that compares random, HTCO (indep), HTCO (2-series) and HTCO for 5\% camouflaging. The RE effort with $\mathrm{HTCO}$ (2-series) is better than other metrics. The same exercise is repeated for three flavors of HDS namely, HDS (2-series), HDS (indep) and HDS, and, two flavors of HTRE namely HTRE (2-series) and HTRE (indep).

The results (Fig 7.3 and Fig 7.4) indicate that series-connected gates perform better in maximizing the RE effort. Fig 7.5 compares the RE effort for the proposed flavors of HTCO, HDS and HTRE metrics for benchmark C888. HTRE(2-series) performs best in maximizing the RE effort. It is interesting to observe that even with small percentage of camouflaging the same level of RE effort can be achieved through careful gate selection metric. This is evident in Fig 7.5 that shows same RE effort for 5\% camouflaging using HTRE(2-series) and 10\% camouflaging using HDS and $15 \%$ camouflaging using random selection. 
From the above results we conclude that the stregnth of camouflaging can be improved by focusing on following approach during gate selection: (i) choose as many dependent gates as possible with given camouflaging budget; (ii) use HTCO, HDS and HTRE metric as guiding to
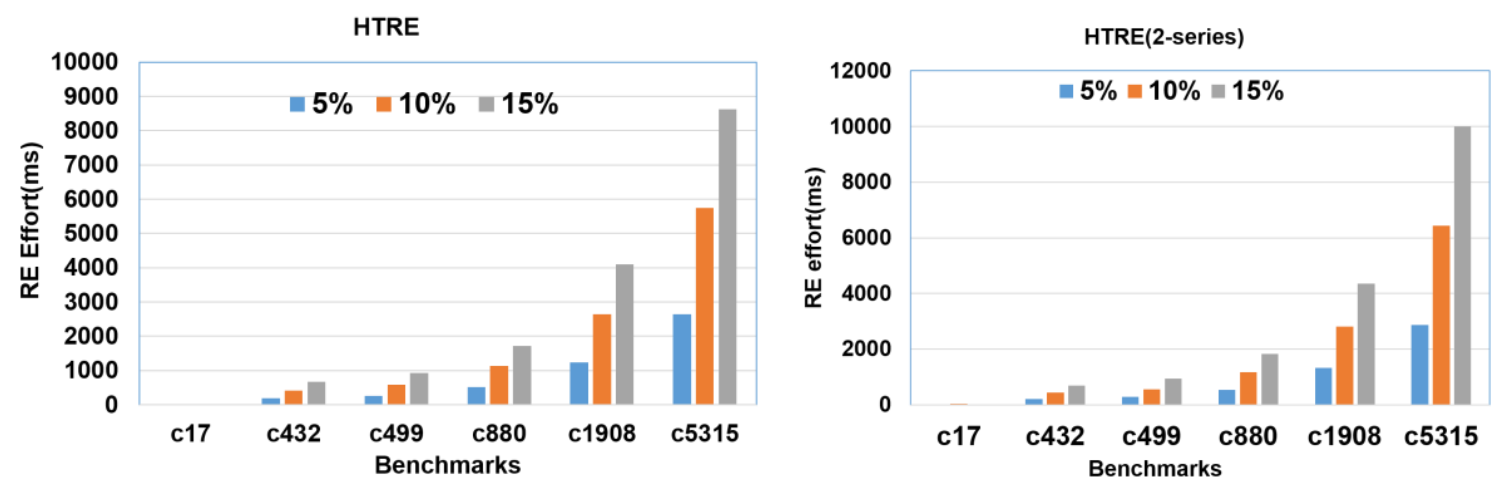

Figure 7.4 RE Effort for HTRE with Pure Gate Selection and 2-Series Dependent Gates.

identify the set of potential gates for camouflaging; (iii) instead of picking independent gates with high metric values, it may be effective to pick dependent gates with low metric values. Future work will involve more detailed analysis of the above factors for gate selection and exploiting test features of ATPG tools to lower the RE effort.

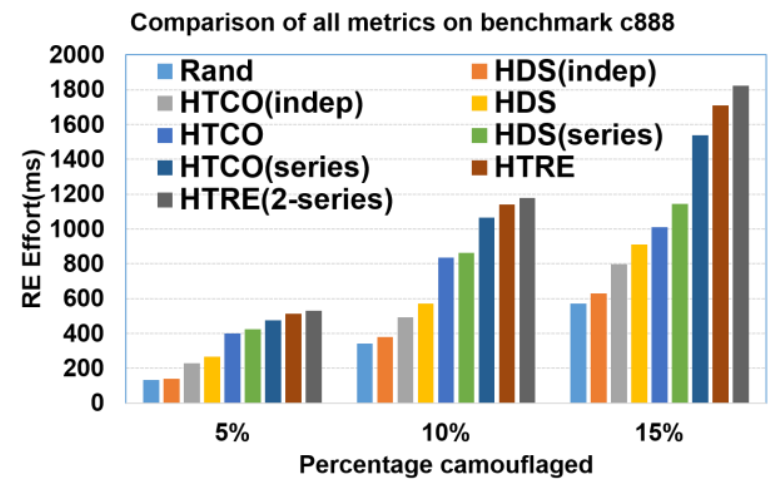

Figure 7.5 Comparison of RE Effort with Various Flavors of HTCO, HDS and HTRE. 


\subsection{Multiplexer RE Results}

Fig 7.6 shows the RE effort for different benchmarks for 2-1 multiplexer. Fig 7.7 shows the RE effort for different type of multiplexers in that plot we can clearly observe that compared to 2-1 mux there is a linear increase in RE effort when we increase the inputs to $4,8,16$.

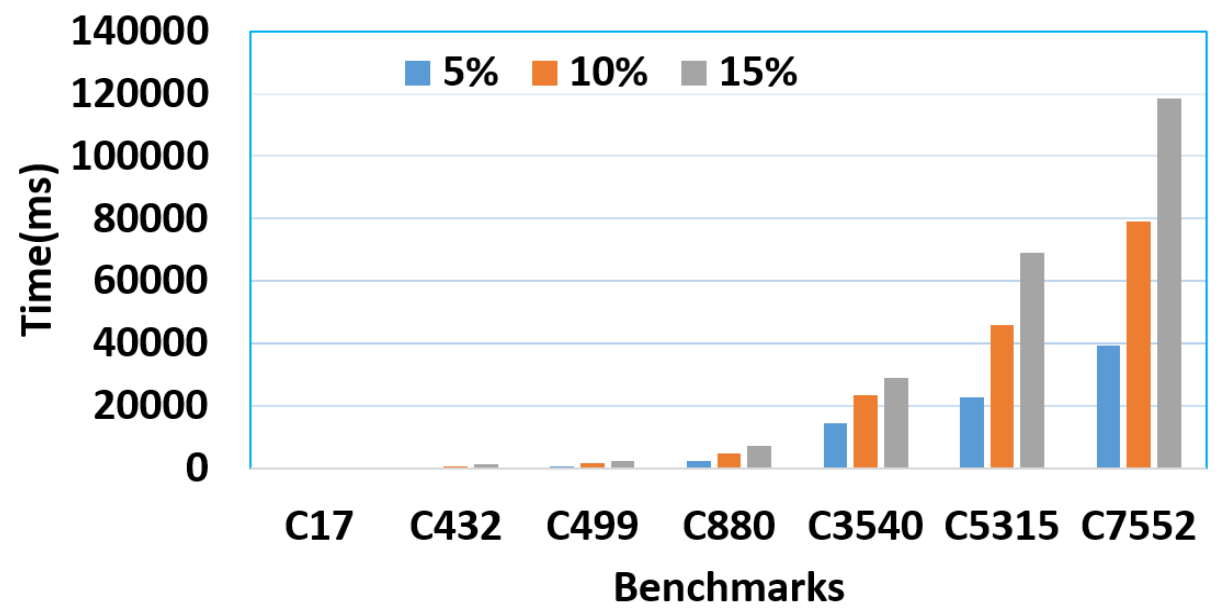

Figure 7.6 RE Effort of Different Benchmarks Replaced with 2-1 Multiplexer.

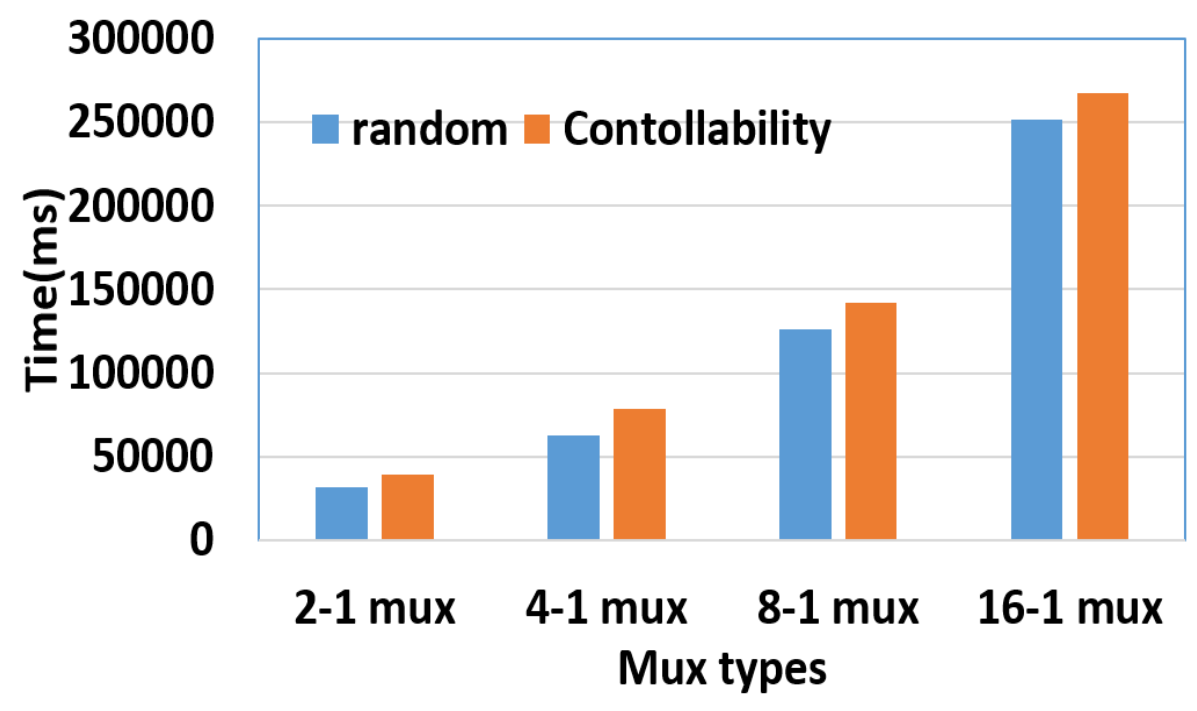

Figure 7.7 RE Effort for Different Multiplexers for 5\% Camouflaging. 


\section{CHAPTER 8}

\section{CONCLUSION}

We propose a method of de-camouflaging the netlist using a commercial ATPG tool. The proposed method is generic and could be employed for single as well as multiple camouflaged gates. We also propose a controllability, observability and HD sensitivity based metrics to select gates for camouflaging. An aggregate RE effort metric is also developed. The effectiveness of the proposed camouflaging methodology is evaulated by comparing the RE efforts. Choice of dependent gates that are selected based on a combination of controllability, observability and HD sensitivity based metric shows best performance for maximizing RE effort. 


\section{REFERENCES}

[1] Rajendran, Jeyavijayan, Michael Sam, Ozgur Sinanoglu, and Ramesh Karri. "Security analysis of integrated circuit camouflaging." In Proceedings of the 2013 ACM SIGSAC conference on Computer \& communications security, pp. 709-720. ACM, 2013.

[2] Chipworks, "Intel’s 22-nm Tri-gate Transistors Exposed", http://www.chipworks.com/blog/technologyblog/2012/04/23/intels-22-nm-trigate-transistorsexposed/, 2012.

[3] Torrance, Randy, and Dick James. "The state-of-the-art in semiconductor reverse engineering." In Proceedings of the 48th Design Automation Conference, pp. 333-338. ACM, 2011.

[4] Almasi, Darya. "ENHANCING HARDWARE SECURITY USING SPIN TRANSFER TORQUE LOGIC." PhD diss., San Francisco State University, 2015.

[5] Chipworks, "Reverse engineering software," http://www.chipworks.com/en/technicalcompetitiveanalysis/resources/reverseengineering- software.

[6] Degate, http://www.degate.org/documentation/.

[7] SEMI, "Innovation is at risk as semiconductor equipment and materials industry loses up to \$4 billion annually due to IP infringement," www.semi.org/en/Press/P043775, 2008.

[8] SypherMedia, "Syphermedia library circuit camouflage technology," http://www.smi.tv/solutions.htm.

[9] ATPG TOOLS, https://en.wikipedia.org/wiki/Automatic_test_pattern_generation. 


\section{[10] Synopsys Tetramax}

http://www.synopsys.com/Tools/Implementation/RTLSynthesis/Test/Pages/TetraMAXATPG.as px

[11] Imeson, Frank, Ariq Emtenan, Siddharth Garg, and Mahesh Tripunitara. "Securing computer hardware using 3D integrated circuit (IC) technology and split manufacturing for obfuscation." In Presented as part of the 22nd USENIX Security Symposium (USENIX Security 13), pp. 495510. 2013.

[12] Baukus, James P., Lap-Wai Chow, and William M. Clark Jr. "Method and apparatus using silicide layer for protecting integrated circuits from reverse engineering." U.S. Patent 6,117,762, issued September 12, 2000.

[13] Chow, Lap-Wai, James P. Baukus, and William M. Clark Jr. "Integrated circuits protected against reverse engineering and method for fabricating the same using an apparent metal contact line terminating on field oxide." U.S. Patent 7,294,935, issued November 13, 2007.

[14] Chakraborty, Rajat Subhra, and Swarup Bhunia. "Hardware protection and authentication through netlist level obfuscation." In Proceedings of the 2008 IEEE/ACM International Conference on Computer-Aided Design, pp. 674-677. IEEE Press, 2008.

[15] Chakraborty, Rajat Subhra, and Swarup Bhunia. "Security against hardware Trojan attacks using key-based design obfuscation." Journal of Electronic Testing 27, no. 6 (2011): 767-785.

[16] Chakraborty, Rajat Subhra, and Swarup Bhunia. "Security against hardware Trojan through a novel application of design obfuscation." In Proceedings of the 2009 International Conference on Computer-Aided Design, pp. 113-116. ACM, 2009. 
[17] Rajendran, Jeyavijayan, Youngok Pino, Ozgur Sinanoglu, and Ramesh Karri. "Security analysis of logic obfuscation." In Proceedings of the 49th Annual Design Automation Conference, pp. 83-89. ACM, 2012.

[18] El Massad, Mohamed, Siddharth Garg, and Mahesh V. Tripunitara. "Integrated Circuit (IC) Decamouflaging: Reverse Engineering Camouflaged ICs within Minutes." In NDSS. 2015.

[19] Tools, Synopsys Test. "TetraMAX ATPG." (2003).

[20] Iyengar, Anirudh, and Swaroop Ghosh. "Threshold Voltage-Defined Switches for Programmable Gates." GOMACTech, 2015.

[21] Bushnell, Michael, and Vishwani D. Agrawal. Essentials of electronic testing for digital, memory and mixed-signal VLSI circuits. Vol. 17. Springer Science \& Business Media, 2000.

[22] Dhare, Vaishali H., and Usha Mehta. "Object Oriented Implementation of Combinational Controllability and Observability Algorithms." International Journal on Electronics Engineering: Kurukshetra, Hariyana Volume2, Number-1 (2010): 93-97.

[23] Nirmala, Ithihasa Reddy, Deepak Vontela, Swaroop Ghosh, and Anirudh Iyengar. "A novel threshold voltage defined switch for circuit camouflaging." In 2016 21th IEEE European Test Symposium (ETS), pp. 1-2. IEEE, 2016.

[24] Deepak reddyVontela and Swaroop Ghosh "Methodologies to Exploit ATPG Tools for Decamouflaging", GOMACTECH, 2016.

[25] Maksim Jenihhin's Iscas85 Benchmark Circuits, http://www.pld.ttu.ee/ maksim/benchmarks/iscas85/verilog/. 


\section{APPENDIX A \\ COPYRIGHT PERMISSIONS}

Below is the permissions for the use of material in Chapter 1.

\section{ASSOCIATION FOR COMPUTING MACHINERY, INC. LICENSE \\ TERMS AND CONDITIONS}

Oct 19,2016

This Agreement between deepakreddy vontela ("You") and Association for Computing Machinery, Inc. ("Association for Computing Machinery, Inc.") consists of your license details and the terms and conditions provided by Association for Computing Machinery, Inc. and Copyright Clearance Center.

\section{All payments must be made in full to CCC. For payment instructions, please see information listed at the bottom of this form.}

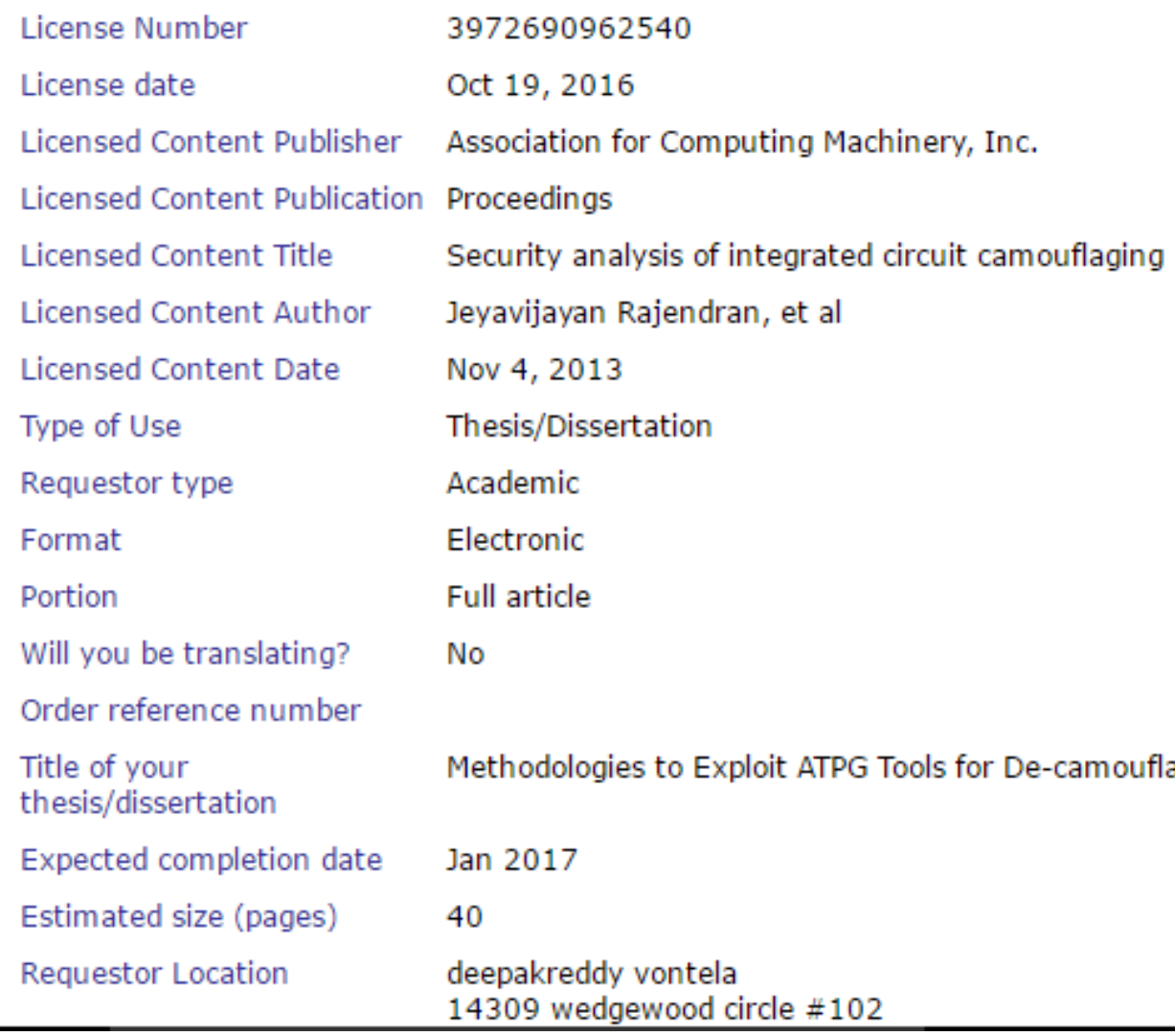




\section{Below is the permission for the use of material in Chapter 4.}

Rightslink@ by Copyright Clearance Center - Google Chrome

https://s100.copyright.com/AppDispatchServlet\#formTop|

Copyright

Clearance

Center

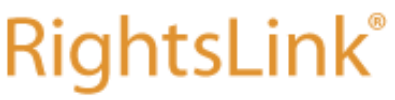

Title:

A novel threshold voltage

Requesting permission

to reuse

content from

an IEEE

publication defined switch for circuit camouflaging

Conference Test Symposium (ETS), 2016

Proceedings: 21th IEEE European

Author: Ithihasa Reddy Nirmala

Publisher: IEEE

Date: May 2016

Copyright (c) 2016, IEEE
If you're a copyright.com user, you can login to RightsLink using your copyright.com credentials. Already a RightsLink user or want to learn more?

Thesis / Dissertation Reuse

The IEEE does not require individuals working on a thesis to obtain a formal reuse license, however, you may print out this statement to be used as a permission grant:

Requirements to be followed when using any portion (e.g., figure, graph, table, or textual material) of an IEEE copyrighted paper in a thesis:

1) In the case of textual material (e.g., using short quotes or referring to the work within these papers) users must give full credit to the original source (author, paper, publication) followed by the IEEE copyright line (c) 2011 IEEE.

2) In the case of illustrations or tabular material, we require that the copyright line (c) [Year of original publication] IEEE appear prominently with each reprinted figure and/or table.

3) If a substantial portion of the original paper is to be used, and if you are not the senior author, also obtain the senior author's approval.

Requirements to be followed when using an entire IEEE copyrighted paper in a thesis:

1) The following IEEE copyright/ credit notice should be placed prominently in the references: (c) [year of original publication] IEEE. Reprinted, with permission, from [author names, paper title, IEEE publication title, and month/year of publication ]

2) Only the accepted version of an IEEE copyrighted paper can be used when posting the paper or your thesis on-line.

3) In placing the thesis on the author's university website, please display the following message in a prominent place on the website: In reference to IEEE copyrighted material which is used with permission in this thesis, the IEEE does not endorse any of [university/educational entity's name goes here]'s products or services. Internal or personal use of this material is permitted. If interested in reprinting/republishing IEEE copyrighted material for advertising or promotional purposes or for creating new collective works for resale or redistribution, please go to http://www.iee.org/publications standards/publications/riahts/rights link.html to learn how to obtain a License from RiahtsLink. 